\title{
Utilitarian and cognitive aspects in the ethnotaxonomy of plants from the Caatinga in two rural communities in Northeastern Brazil
}

\section{Reinaldo Farias Paiva de Lucena ${ }^{1,}$, Patrícia Muniz de Medeiros $^{2}$, Elcida de Lima Araújo ${ }^{3}$, Ângelo G. Chaves Alves $^{3}$, Kallyne Machado Bonifácio ${ }^{1}$, José da Silva Mourão ${ }^{4}$ and Ulysses Paulino de Albuquerque ${ }^{5}$}

\author{
${ }^{1}$ Universidade Federal do Mato Grosso do Sul. Instituto de Biociências. Laboratório \\ de Etnobotânica. Campo Grande-MS, Brasil (CEP 79070-900). Email: \\ reinaldolucena.dr@gmail.com. \\ ${ }^{2}$ Universidade Federal de Alagoas, Centro de Ciências Agrárias. BR-104. Rio \\ Largo-AL, Brasil (CEP 57100-000). \\ ${ }^{3}$ Universidade Federal Rural de Pernambuco. Depatamento de Biologia. Rua Dom \\ Manuel de Medeiros, 97. Dois Irmãos. Recife-PE, Brasil (CEP 52171-900). \\ ${ }^{4}$ Universidade Estadual da Paraíba. Departamento de Biologia. Rua das Baraúnas, \\ 351. Bairro Universitário. Campina Grande-PB, Brasil (CEP 58429-500). \\ 5Universidade Federal de Pernambuco. Centro de Ciências Biológicas, \\ Departamento de Botânica. Av. Reitor Joaquim Amazonas, S/№. Cidade \\ Universitária. Recife-PE, Brasil (CEP 50740-570).
}

\begin{abstract}
This research sought to contribute to plant ethnotaxonomic studies and aimed to describe cognitive and utilitarian aspects used in communities in the semi-arid region of Brazil. The question asked in the interview was: "What plants do you know?" The interviews were conducted with local specialists, using plant names written on cards randomly placed on a table. The informants were asked to organize the cards according to their understanding. Two hundred and one folk generics were recorded in Cachoeira and 185 in Barrocas, both communities located in the Municipality of Soledade (Paraíba). These generics were divided into 65 trees/shrubs, 138 herbs, 10 lianas/creepers, 7 cacti, and 4 bromeliads. A total of 146 monotypic and 24 polytypic folk generic were identified. The life forms were abundant; some of them had already been recorded in the literature but others were recorded for the first time. The morphological and utilitarian aspects were the most used classification criteria. The informants followed no consensus model to organize their classification.
\end{abstract}

Keywords: Biodiversity; Caatinga; Ethnobotany; Ethnotaxonomy.

Received

Octuber 19, 2021

Accept

November 29, 2021

Available online

December 8, 2021

Released

December 31, 2021

Open access

ISSN 2359-1412/RBGAS-2021-0154/2021/8/20/13/1459

Rev. Bras. Gest. Amb. Sustent.

http://revista.ecogestaobrasil.net 
Resumo. Aspectos utilitários e cognitivos na etnotaxonomia de plantas da Caatinga em duas comunidades rurais no Nordeste do Brasil. Resumo. Esta pesquisa buscou contribuir com os estudos etnotaxonômicos das plantas e objetivou descrever aspectos cognitivos e utilitários utilizados em comunidades do semi-árido brasileiro. A pergunta feita na entrevista foi "Quais plantas você conhece?" As entrevistas foram realizadas com especialistas locais, usando nomes de plantas escritos em cartões colocados aleatoriamente em uma mesa. Os informantes foram solicitados a organizar os cartões de acordo com seu entendimento. Duzentos e um genéricos populares foram registrados em Cachoeira e 185 em Barrocas, ambas comunidades localizadas no Município de Soledade (Paraíba). Esses genéricos foram divididos em 65 árvores/arbustos, 138 ervas, 10 lianas/trepadeiras, sete cactos e quatro bromélias. Um total de 146 genéricos monotípicos e 24 politípicos folk genéricos foram identificados. As formas de vida eram abundantes; alguns deles já haviam sido registrados na literatura, mas outros foram registrados pela primeira vez. Os aspectos morfológicos e utilitários foram os critérios de classificação mais utilizados. Os informantes não seguiram um modelo de consenso para organizar sua classificação.

Palavras-chave: Biodiversidade; Caatinga; Etnobotânica; Etnotaxonomia.

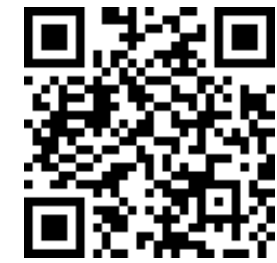

\footnotetext{
ORCID

(1) 0000-0002-1195-4315

Reinaldo Farias Paiva de Lucena

(1) 0000-0002-3835-5232

Patrícia Muniz de

Medeiros

(D) 0000-0002-3379-3540

Elcida de Lima Araújo

(1) 0000-0002-1506-5734

Ângelo G. Chaves Alves

(1) 0000-0002-0767-9226

Kallyne Machado

Bonifácio

(1) 0000-0003-2249-5836

José da Silva Mourão

(C) 0000-0002-8131-8429

Ulysses Paulino de

Albuquerque
}

\section{Introduction}

According to Hennig (1966), biological classifications have formed a general reference system on biological diversity and are real deposits of information. In this sense, the local systems (informal) of folk classification are also important, as they contain a high richness of information on biology, ecology, and ethology of several groups of animals and plants.

Carvalho et al. (2018), based on an ethnotaxonomy study involving shark fishermen in Northeastern Brazil, showed that the details in the identification and local classification of this fishery resource may contribute to the management, conservation, and sustainable use of the species recorded in the region.

Amorim (1997) argued that the lack of people's knowledge of biological diversity generates a limited number of classification levels, compared to those produced by technical knowledge. However, little knowledge of the ethnobiology literature by some researchers do not allow them to understand that the few inclusion levels in the folk classification do not necessarily result from the lack of knowledge of local and traditional cultures, but because these often represent a comprehension culturally based on the natural world.

There are cases in which the disappearance of the local name of a taxon in a certain social group may lead to the disappearance of cultural connections, with consequences in the ethnoecological knowledge. For example, the identification and classification of some insects by people from San Miguel el Grande, Mexico, was not possible because they were 
little culturally relevant species and due to the influence of the Spanish language (Aparicio et al., 2018).

Comparing the detected patterns in folk systems with the formal biological taxa (academics) to which they refer would be, according to Berlin (1992), one of the steps to understand the analogies between the two classification systems. Thus, ethnotaxonomic studies generally represent comparison attempts between the classification systems of different studied cultures, as well as between these and the so-called scientific classifications.

Some studies on folk classification have been performed over the years aiming to understand how local populations have classified and related to the environment, especially to plants and animals (Berlin et al., 1968; Berlin, 1973; Bulmer, 1974, Berlin, 1976; Hunn, 1982; Hays, 1983; Berlin, 1992; Ellen, 1993; Clément, 1995; Carrara, 1997; Atran, 1998; Jinxiu et al., 2004; Mourão et al., 2006; Farias and Alves, 2007; Souza and Begossi, 2007). Some of the main conclusions refer to an apparent similarity in the way how different people taxonomically classify and organize living beings. For example, artisanal fishermen, on the coasts of Pernambuco and Ceará (Northeastern Brazil), attributed similar names to fish species. This possibly suggests a geographical continuity in the nomenclature pattern along the Brazilian coast (Pinto et al., 2016).

The populations use morphological, ecological, utilitarian, and behavioral aspects in the taxonomic organization (Mourão et al., 2006). Economic criteria have also been recorded, from which the same species can be named according to its local importance (Jinxiu et al., 2004).

Since the beginning of this century, ethnotaxonomic approaches concerning the animal universe have predominated in Brazil (Mourão, 2000; Mourão and Nordi 2002a,b; Araújo, 2005; Mourão et al., 2006; Mourão and Montenegro, 2006; Mourão and Nordi, 2006; Farias and Alves, 2007; Souza and Begossi, 2007; Pinto et al., 2016; Braga, 2017; Carvalho et al. 2018), including few studies on plant ethnotaxonomy (Hanazaki et al., 2006; Haverroth, 2007).

In addition to the lack of studies aimed to understand the relationship between people and plants, few of them have a conservationist approach. Jinxiu et al. (2004) found a high similarity between scientific classification and folk classification (classification performed by local or traditional populations) in the ethnicity "Dai" (Southwest China) and argued that the ethnobiological taxonomy may be used in rapid survey studies on local biodiversity, detecting species in decline that deserve conservation attention.

Given the above, this research sought to contribute to strengthening plant ethnotaxonomic studies and aimed to describe the cognitive and utilitarian aspects adopted by residents of rural communities in the semi-arid region of Brazil. Furthermore, it sought to analyze comparatively and understand how the local ethnotaxonomy has been built, assuming that its comprehension, by scientists and decision-makers, may contribute to providing information for the development of conservation plans for the local diversity, because such ethnotaxonomy may be used in rapid biodiversity surveys. Also, it was verified if there was any relationship between the plant classification criteria used by the informants and the priorities indicated by Lucena et al. (2013) for the conservation of woody species in that same region. Moreover, according to our knowledge, this is the first study on this subject in the semi-arid region of State of Paraíba, Northeastern Brazil.

\section{Material and methods}

\section{Regional context and study area}

This study was conducted in a semi-arid environment in Northeastern Brazil, specifically in the rural communities of Barrocas and Cachoeira, both located in the Municipality of Soledade, Paraíba State (Figure 1). Soledade was founded by Decree No. 
171, of September 24th, 1885. It is located in the "Agreste" Mesoregion and "Curimataú Ocidental" Microregion ( $7^{\circ} 03^{\prime} 26^{\prime \prime} \mathrm{S}$ and $36^{\circ} 21^{\prime} 46^{\prime \prime} \mathrm{W}$ ), at 521 meters above the sea level and $180 \mathrm{~km}$ away from João Pessoa, the state capital (SEBRAE, 1998). This municipality has a territorial area of 560,062 $\mathrm{km}^{2}$ and an estimated population of 13,128 inhabitants. It has 24,982 hectares of areas covered with native vegetation (SEBRAE, 1998).

Soledade has a hot semi-arid climate (BShs' according to Köppen), annual rainfall around $300 \mathrm{~mm}$, short rainy season, and up to eleven months of drought (Geographical Atlas from State of Paraíba, 1985). This town has one of the lowest precipitation rates in the Northeast Region of Brazil (SEBRAE, 1998). The local vegetation is hyperxerophilous shrub-arboreal, with a high density of cacti, which is currently noticeably predominant, due to the massive tree deforestation for energy production, such as firewood and charcoal to be used in homes (SEBRAE, 1998). The soil is predominantly halomorphic, with a high salinity level, interfering with the development of projects for the use of subsurface water (SEBRAE, 1998).

\section{Characteristics of the study communities}

The community of Barrocas, during the fieldwork period, accounted for 12 inhabited houses, predominantly private rural properties, with no clustered houses. Corn and bean were the predominant cultures in the community, mostly for subsistence. Cattle, goat, and sheep rearing was the most recorded livestock practice, with a low frequency of poultry and swine. The study area (Caatinga) comprised different phytophysiognomies with the presence of primary and secondary vegetation areas, and regeneration areas, represented by abandoned cultivation and pasture areas.

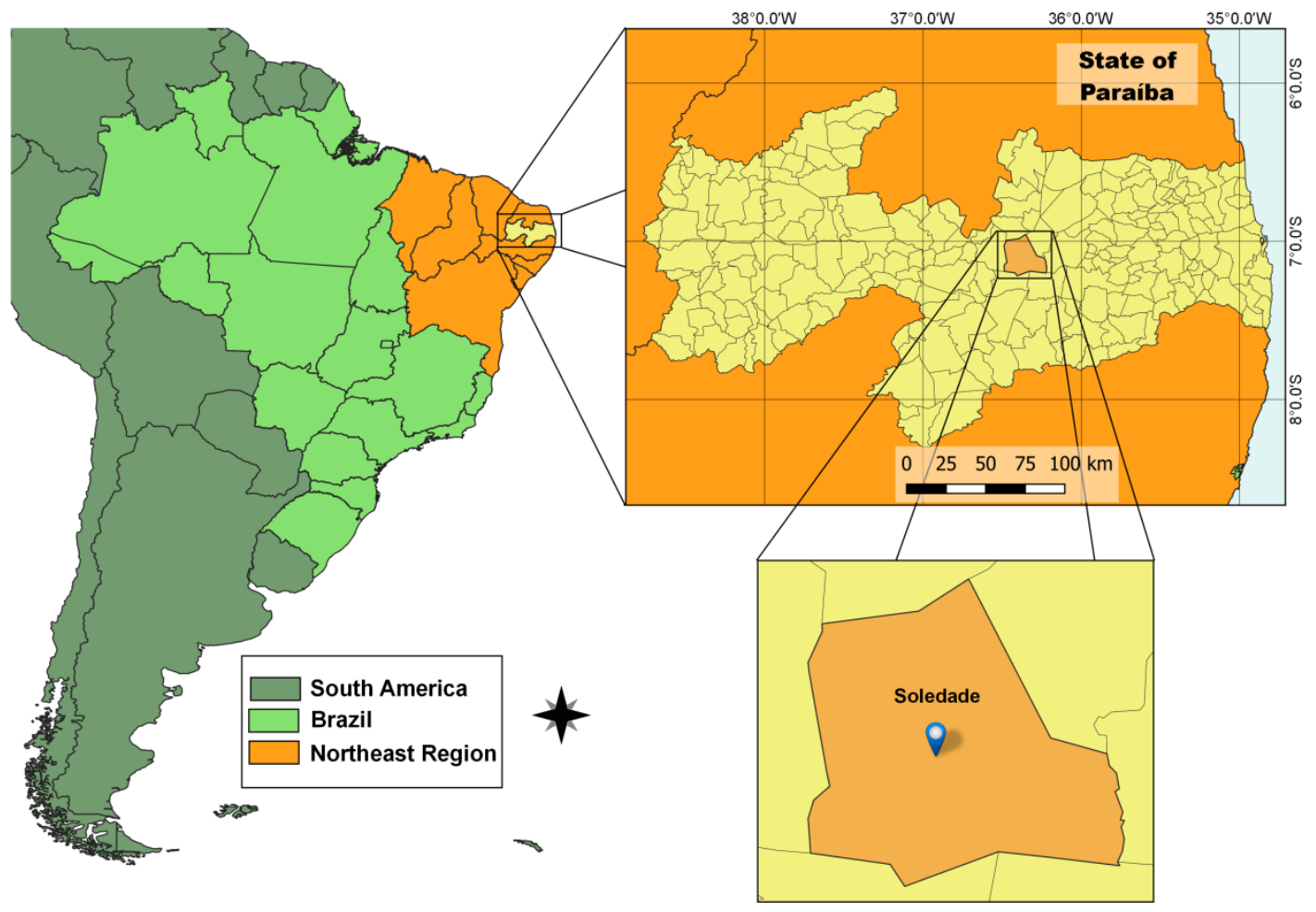

Figure 1. Location of the study area in the Municipality of Soledade (Paraíba, Northeastern Brazil). 
The areas bordered by rivers, streams, and ponds were composed of arboreal vegetation, and in other areas, there were shrub/herbaceous-sized species spaced from each other. A predominance of cacti and bromeliads was recorded, which had been widely used for animal feed. The area was crossed by intermittent rivers, which had a large volume of water running during "winter time" (local name for rainy season), mainly in March and June, with the occurrence of sporadic flooding. The areas bathed by rivers had been widely used for the cultivation of cereal and forage species, such as those belonging to the family Poaceae.

In Cachoeira, differently from Barrocas, there were 18 inhabited houses, particularly like a village, with clusters of houses distributed throughout the community, bordering the local road. The territorial area in this community had been divided between two families, which had given the lands so that other residents could build their homes. Another particularity is that the native vegetation fragment had already been used by all residents, especially because of their kinship ties. Such use may have led certain species to local extinction risks, as Anadenanthera colubrina (Vell.) Brenan (angico), according to the oldest residents, had been widely used for charcoal production and sold to tanneries in Campina Grande (regional urban center at $60 \mathrm{~km}$ away from Soledade).

Barrocas and Cachoeira have similarities regarding agropastoral activities, such as a predominance of corn and beans cultivation and goat and bovine rearing. To access health units, shopping centers, and schools, people from both communities need to go to the urban center of Soledade or other nearby communities, such as the district of Bom Sucesso.

\section{Local botanical knowledge survey}

Before starting the interviews, a conversation was held with each informant, explaining the aim of the study. Afterward, they were asked to sign the consent form required by the National Health Council through the Research Ethics Committee (Resolution 196/1996). Then, the data related to the local botanical knowledge were collected through semi-structured interviews, conducted between 2006 and 2008, with all family heads (men or women) (Albuquerque et al., 2010) in the two communities.

To obtain the date, the following question was asked in the interview: "What plants do you know?" From this question, all folk generics were surveyed (Berlin 1996), including the exotic species which were used in medicine, ornamentation, and animal feeding. All woody, cactus, bromeliad, herbaceous, creeper, liana, and epiphyte species were included in the list.

Through the list of folk generics, a database was prepared and used to make identification cards of folk generics and specifics. Subsequently, these cards were used by the specialists to group the plant species. The criteria used to choose these informants were similar to those used for "native specialists" (Marques, 2001), i.e., all family heads of both communities who were considered experts of the local flora. Such specialists were identified from informal conversations with residents. Each card was made using cardboard, on which each folk generics and specifics were written. Five local specialists were identified in Barrocas (four men and one woman) and 12 in Cachoeira (seven men and five women).

The interviews with the local specialists were performed as follows: all cards were randomly placed on a table; then, the informants were asked to organize the cards containing the plant names according to their understanding. Two hundred one cards were shown in Cachoeira and 185 in Barrocas.

Each specialist was informed that plants could be separated (alone) if their characteristics did not correspond to any group, and that unknown plant cards could be classified into another group. This step was performed with each informant separately. After finishing this first grouping, which resulted in "large groups", the informants were 
asked if they could form smaller groups into those previously formed, creating subgroups within each large group. The names of the plants and their respective groups and subgroups were recorded in a notebook. Subsequently, the specialists were asked about the reason for forming each group, in order to infer the classification criteria adopted by each one.

To perform an analysis from a conservationist point of view of woody plants in the communities, using local classification, some species indicated by Lucena et al. (2013) as worthy of conservation, in a research conducted in the same study area, were selected. In this analysis, it was sought to identify species that shared the same utilitarian criteria of those indicated by Lucena et al. (2013) as priorities for conservation, which would be grouped in the same group in the classification performed by the specialists. Identifying (or not) such species, it would be possible to propose their shared use, following a sustainable management system, which would reduce the exploitation pressure on the species indicated to immediate conservation.

The following species were selected: Aspidosperma pyrifolium Mart. (pereiro), Commiphora leptophloeos (Mart.) J. B. Gillet. (umburana), Croton blanchetianus Baill. (marmeleiro), Myracrodruon urundeuva Allemão (aroeira), Poincianella pyramidalis Tul. (catingueira), Sideroxylon obtusifolium (Roem. \& Schult.) T. D. Penn. (quixabeira), Ximenia americana L. (ameixa), and Schinopsis brasiliensis Engl. (baraúna). They were selected through triangulation of results obtained by different research methods, such as the use value, conservation priority index, and inventory in situ (Lucena et al., 2013). This triangulation resulted in a list in descending order of the more vulnerable species in the region, from which the abovementioned species were chosen.

\section{Data analysis}

The groups were analyzed according to the criteria and justifications proposed by the local specialists. These criteria were compared with those found in the literature, in addition to confirming through specialized literature the names attributed to the obtained categories. In the comparison of the local classification with the formal one (scientific), the hierarchical categories of taxonomic inclusion proposed by Berlin (1992) were considered, as follows: unique beginner (kingdom), life form, intermediate, generic, specific, and varietal (variety). The context of the generics was analyzed dividing them into monotypic (when it had only one member, which could be either simple or compound name), and polytypic (when it was composed by two or more members).

Another comparison between the local and scientific classifications was based on taxonomic correspondence, which can be (1) correspondence 1:1, in which a folk generic corresponded to one species; (2) over-differentiation, in which two or more folk generics corresponded to one species; and (3) sub-differentiation, which was divided into two subtypes: sub-differentiation No. 1, when one folk generic represented two or more species of the same genus, and sub-differentiation No. 2 when it was evidenced a correlation of a folk generic with two or more species of a different genus.

For analyzing and representing the groups formed by the local specialists, it was used the Venn Diagram adopted by Berlin (1992) and the Pile Sort Multidimensional Scaling in the ANTHROPAC software (Analytic Technologies \& Medical Decision Logic, Inc. version 1.0). This software was used to analyze the consensus degree and cultural competence of the specialists, regarding their classifications, in addition to assessing the proximity regarding the classification criteria used by them. The data analysis of the species, classified by Lucena et al. (2013) as worthy of conservation efforts, was performed based on the groupings obtained from the abovementioned Scaling (Analytic Technologies \& Medical Decision Logic, Inc. version 1.0).

For botanical identification, plants were collected through the guided tour method, with the aid of woodsmen and local specialists, after the general interviews with the 
community. Afterward, they were identified at a species level, when it was possible, using analytical keys and by comparison with the material deposited in the Herbarium Vasconcelos Sobrinho (PEUFR), of the Federal Rural University of Pernambuco, as well as consulting specialists (scientists).

PEUFR: Herbário Vasconcelos Sobrinho from the Federal Rural University of Pernambuco. * Herbarium of the Agricultural Research Institute of Pernambuco - IPA. ** UFP - Herbário Geraldo Mariz of the Federal University of Pernambuco. ${ }^{* * *}$ Herbarium Jaime Coêlho de Moraes (EAN) of the Federal University of Paraíba, at the Center for Agricultural Sciences. Collector: Lucena, R. F. P. Hebrew PEUFR Collector: Almeida, C. Hebário UFP - Geraldo Mariz. Federal University of Pernambuco. Collector: Trajano, A. Hebário IPA Dárdano de Andrade Lima. Pernambuco Agricultural Research Corporation.

\section{Results}

Two hundred one folk generics were recorded in Cachoeira and 185 in Barrocas, among which, 158 were shared by both communities. The generics were divided into 65 trees/shrubs, 138 herbaceous, 10 lianas/creepers, 7 cacti, and 4 bromeliads (Table 1). The classification made in both communities consisted of four levels of hierarchical structure, proposed by Berlin (1992), and no intermediate and varietal levels were identified (Figure 2). One hundred forty-six monotypic and 24 polytypic generics were identified among those recorded and named by the informants. From the generics folk which were registered and named by the informants, 146 were monotypic and 24 polytypic.

Table 1. Generic folks cited by local specialists in the communities of Barrocas and Cachoeira, located in the Municipality of Soledade (Paraíba, Northeastern Brazil). Classification criteria: E ecological, L - linguistic, M - morphological, MR - magic-religious, NU - non-utilitarian, S - separated (alone), and U - utilitarian. Life forms: T - tuberous, G - grass, L - liana, Th - thorny, Lb - large.

\begin{tabular}{|c|c|c|c|c|}
\hline $\begin{array}{c}\text { Vernacular } \\
\text { name }\end{array}$ & Scientific name & Family & $\begin{array}{c}\text { Classification } \\
\text { criteria }\end{array}$ & $\begin{array}{c}\text { Life } \\
\text { form }\end{array}$ \\
\hline Agave & Agave sisalana Perrine & Agavaceae & E; M; U & $\begin{array}{l}\text { E; Mg; } \\
\text { Mm; } \\
\text { Mp }\end{array}$ \\
\hline Alecrim & Rosmarinus officinalis $\mathrm{L}$. & Lamiaceae & $\mathrm{M} ; \mathrm{U}$ & $\mathrm{C} ; \mathrm{Mp}$ \\
\hline $\begin{array}{ll}\text { Alecrim de } \\
\text { serrote }\end{array}$ & Portulaca elatior Mart. ex Rohrb. & Portulacaceae & M; NU; U & $\begin{array}{l}\text { C; Mm; } \\
\mathrm{Mp}\end{array}$ \\
\hline $\begin{array}{l}\text { Alfazema } \\
\text { braba }\end{array}$ & Hyptis suaveolens (L.) Poit. & Lamiaceae & L; M; U & $\mathrm{Mp}$ \\
\hline Algaroba & Prosopis juliflora (Sw.) DC. & Fabaceae & $\mathrm{MU}$ & $\mathrm{Mg}$ \\
\hline $\begin{array}{l}\text { Algodão de } \\
\text { cera }\end{array}$ & Calotropis procera (Willd.) R. Br. & Asclepiadaceae & $\mathrm{M} ; \mathrm{U}$ & $\begin{array}{l}\text { Mg; } \\
\text { Mm }\end{array}$ \\
\hline $\begin{array}{l}\text { Algodão } \\
\text { preto }\end{array}$ & Gossypium hirsutum L. & Malvaceae & $\mathrm{M}$ & $\mathrm{C} ; \mathrm{Mm}$ \\
\hline $\begin{array}{l}\text { Amarra } \\
\text { cachorro }\end{array}$ & Jacquemontia bahiensis O'Donell & Convolvulaceae & $\mathrm{M} ; \mathrm{U}$ & $\begin{array}{l}\text { Mp; } \\
\text { Mr; R }\end{array}$ \\
\hline Ameixa & Ximenia americana $\mathrm{L}$. & Olacaceae & $\mathrm{M} ; \mathrm{U}$ & $\begin{array}{l}\mathrm{A} ; \mathrm{Mg} ; \\
\mathrm{Mm}\end{array}$ \\
\hline Ameixa & PtilochaetabahiensisTurcz. & Malpighiaceae & $\mathrm{M} ; \mathrm{U}$ & $\begin{array}{l}\text { A; Mg; } \\
\text { Mm }\end{array}$ \\
\hline $\begin{array}{l}\text { Amor de } \\
\text { veio }\end{array}$ & Mentzelia aspera L. & Loasaceae & $\mathrm{U}$ & $\mathrm{Mp} ; \mathrm{Mr}$ \\
\hline
\end{tabular}

Rev. Bras. Gest. Amb. Sustent., 2021, vol. 8, n. 20, p. 1459-1488. 
Table 1. Continued.

\begin{tabular}{|c|c|c|c|c|}
\hline $\begin{array}{c}\text { Vernacular } \\
\text { name }\end{array}$ & Scientific name & Family & $\begin{array}{c}\text { Classification } \\
\text { criteria }\end{array}$ & $\begin{array}{c}\text { Life } \\
\text { form }\end{array}$ \\
\hline Anador & Artemisia vulgaris L. & Asteraceae & $\mathrm{M} ; \mathrm{U}$ & $\mathrm{Mp}$ \\
\hline Angico & $\begin{array}{l}\text { Anadenanthera colubrina (Vell.) } \\
\text { Brenan }\end{array}$ & Fabaceae & $\mathrm{M} ; \mathrm{U}$ & $\mathrm{Mg}$ \\
\hline Anil & Indigofera suffruticosa Mill. & Fabaceae & $\mathrm{M} ; \mathrm{NU} ; \mathrm{U}$ & $\mathrm{Mp}$ \\
\hline Aroeira & Myracrodruon urundeuva Allemão & Anacardiacea & $\mathrm{M} ; \mathrm{U}$ & $\mathrm{A} ; \mathrm{Mg}$ \\
\hline $\begin{array}{l}\text { Arruda; } \\
\text { Arruda da } \\
\text { folha azul }\end{array}$ & Ruta graveolens L. & Rutaceae & $\mathrm{L} ; \mathrm{M} ; \mathrm{U}$ & $\mathrm{Mp}$ \\
\hline Avanço & $\begin{array}{l}\text { Froelichia humboldtiana (Roem. \& } \\
\text { Schult.) Seub. }\end{array}$ & Amaranthaceae & $\mathrm{M} ; \mathrm{U}$ & $\begin{array}{l}\text { C; Mp; } \\
\text { Mr; R }\end{array}$ \\
\hline Avanço & Alternanthera brasiliana (L.) Kuntz. & Amaranthaceae & $\mathrm{M} ; \mathrm{U}$ & $\begin{array}{l}\text { C; Mp; } \\
\text { Mr; R }\end{array}$ \\
\hline Bamburrar & Blainvillea acmella (L.) Philipson & Asteraceae & $\mathrm{M} ; \mathrm{U}$ & $\begin{array}{l}\text { C; Mm; } \\
\text { Mp; } \\
\text { Mr; }\end{array}$ \\
\hline Barauna & Schinopsis brasiliensis Engl. & Anacardiaceae & $\mathrm{M} ; \mathrm{U}$ & $\mathrm{A} ; \mathrm{Mg}$ \\
\hline $\begin{array}{l}\text { Barba de } \\
\text { bode }\end{array}$ & Cyperus uncinulatus Schrad. ex Nees & Cyperaceae & $\mathrm{M} ; \mathrm{U}$ & $\begin{array}{ll}\mathrm{C} ; \mathrm{Mp} ; \\
\mathrm{R}\end{array}$ \\
\hline Barriguda & Ceiba glaziovii( Kuntze) K. Schum. & Malvaceae & $\mathrm{M} ; \mathrm{U}$ & $\mathrm{A} ; \mathrm{Mg}$ \\
\hline $\begin{array}{l}\text { Batata de } \\
\text { purga }\end{array}$ & Operculina macrocarpa (L.) Urb. & Convovulaceae & $\mathrm{M} ; \mathrm{U}$ & $\begin{array}{l}\text { B; C; } \\
\text { Mm; } \\
\text { Ms; R }\end{array}$ \\
\hline Beduega & Portulaca oleracea L. & Portulacaceae & L; M; U & $\mathrm{Mp} ; \mathrm{Mr}$ \\
\hline $\begin{array}{l}\text { Bom dia } \\
\text { branco }\end{array}$ & Cantharanthus roseus(L.) G. Don & Apocynaceae & $\mathrm{U}$ & $\mathrm{Mp}$ \\
\hline $\begin{array}{l}\text { Bom dia } \\
\text { vermelho }\end{array}$ & Cantharanthus roseus (L.) G. Don & Apocynaceae & $\mathrm{U}$ & $\mathrm{Mp}$ \\
\hline Boa noite & Cantharanthus roseus (L.) G. Don & Apocynaceae & $\mathrm{M} ; \mathrm{U}$ & $\mathrm{Mp}$ \\
\hline Boldo & Peumus boldus Mol. & Monimiaceae & $\mathrm{U}$ & $\mathrm{Mp}$ \\
\hline Bom nome & Monteverdia rigida (Mart.) Biral & Celastraceae & $\mathrm{M} ; \mathrm{U}$ & $\begin{array}{l}\mathrm{A} ; \mathrm{Mg} ; \\
\mathrm{Mm}\end{array}$ \\
\hline Bredo & Trianthema portulacastrum L. & Aizoaceae & L; M; U & $\mathrm{Mp} ; \mathrm{Mr}$ \\
\hline $\begin{array}{ll}\text { Bredo de } \\
\text { Espinho; } \\
\text { Bredo de } \\
\text { porco } & \\
\end{array}$ & Amaranthus cruentus L. & Amaranthaceae & L; M & $\mathrm{Mp} ; \mathrm{Mr}$ \\
\hline $\begin{array}{l}\text { Burra } \\
\text { leiteira }\end{array}$ & Sapium lanceolatum L. & Euphorbiaceae & $\mathrm{M} ; \mathrm{U}$ & $\mathrm{A} ; \mathrm{Mg}$ \\
\hline Cabacinha & Luffa operculata Cong. & Cucurbitacea & $\mathrm{M} ; \mathrm{U}$ & Mp; R \\
\hline $\begin{array}{l}\text { Cabeça de } \\
\text { nêgo }\end{array}$ & Wilbrandia sp. & Cucurbitaceae & E; M; U & $\begin{array}{l}\mathrm{B} ; \quad \mathrm{C} \text {; } \\
\mathrm{Mm} ; \\
\mathrm{Mp} ; \\
\mathrm{Ms} ; \mathrm{R}\end{array}$ \\
\hline Caibeira & $\begin{array}{l}\text { Tabebuia aurea (Silva Manso) Benth. } \\
\text { \& Hook.f. ex S. Moore }\end{array}$ & Bignoniaceae & M & $\mathrm{A} ; \mathrm{Mm}$ \\
\hline $\begin{array}{l}\text { Cama de } \\
\text { amancebado }\end{array}$ & Gomphrena demissa Mart. & Amaranthaceae & $\mathrm{M} ; \mathrm{NU} ; \mathrm{U}$ & $\mathrm{Mp} ; \mathrm{Mr}$ \\
\hline $\begin{array}{ll}\text { Cana } & \mathrm{de} \\
\text { macaco } & \\
\end{array}$ & Maranta divaricata Roscoe & Maranthaceae & L; M & $\mathrm{Mm}$ \\
\hline Canafistula & $\begin{array}{l}\text { Senna martiana (Benth.) H. S. Irwin } \\
\text { Barneby }\end{array}$ & Fabaceae & $\mathrm{M} ; \mathrm{U}$ & $\begin{array}{l}\mathrm{Mg} ; \\
\mathrm{Mm}\end{array}$ \\
\hline
\end{tabular}


Table 1. Continued.

\begin{tabular}{|c|c|c|c|c|}
\hline $\begin{array}{c}\text { Vernacular } \\
\text { name }\end{array}$ & Scientific name & Family & $\begin{array}{c}\text { Classification } \\
\text { criteria }\end{array}$ & $\begin{array}{l}\text { Life } \\
\text { form }\end{array}$ \\
\hline $\begin{array}{l}\text { Canafistula } \\
\text { braba }\end{array}$ & $\begin{array}{l}\text { Senna macranthera (Collad.) H. S. } \\
\text { Irwin \& Barneby var. pudibunda } \\
\text { (Benth.) H. S. Irwin \& Barneby }\end{array}$ & Fabaceae & $\mathrm{M} ; \mathrm{U}$ & $\mathrm{Mg}$ \\
\hline $\begin{array}{l}\text { Canafistula } \\
\text { do mato }\end{array}$ & $\begin{array}{l}\text { Senna spectabilis (DC) H. S. Irwin \& } \\
\text { Barneby var. excelsa (Schrad.) H. S. } \\
\text { Irwin \& Barneby }\end{array}$ & Fabaceae & M & $\begin{array}{l}\mathrm{A} ; \mathrm{Mg} ; \\
\mathrm{Mm}\end{array}$ \\
\hline $\begin{array}{l}\text { Canela de } \\
\text { ema }\end{array}$ & Thiloa glaucocarpa (Mart.) Eichler & Combretaceae & M & $\begin{array}{l}\text { Mg; } \\
\text { Mm }\end{array}$ \\
\hline $\begin{array}{l}\text { Capim } \\
\text { belota }\end{array}$ & Chloris orthonoton Döll & Poaceae & $\mathrm{U}$ & $\mathrm{Mm}$ \\
\hline $\begin{array}{l}\text { Capim } \\
\text { panasco }\end{array}$ & $\begin{array}{l}\text { Anthephora hermaphrodita (L.) } \\
\text { Kuntze }\end{array}$ & Poaceae & $\mathrm{M} ; \mathrm{U}$ & C; Mp \\
\hline $\begin{array}{l}\text { Capim pé de } \\
\text { galinha }\end{array}$ & Dactyloctenium aegyptium (L.) Willd. & Poaceae & $\mathrm{M} ; \mathrm{NU} ; \mathrm{U}$ & $\mathrm{C} ; \mathrm{Mp}$ \\
\hline Capim santo & Cymbopogon citratus (DC.) Stapf & Poaceae & $\mathrm{U}$ & $\mathrm{Mp}$ \\
\hline Capitão & Gomphrena basilanata Suess. & Amaranthaceae & $\mathrm{L} ; \mathrm{U}$ & $\mathrm{Mp}$ \\
\hline Cardeiro & Cereus jamacaru DC. & Cactaceae & $\mathrm{L} ; \mathrm{M} ; \mathrm{U}$ & $\mathrm{E} ; \mathrm{Mg}$ \\
\hline Cardo santo & Argemone mexicana $\mathrm{L}$. & Papaveraceae & E; L; NU & $\mathrm{Mp}$ \\
\hline Carnaúba & $\begin{array}{llll}\begin{array}{l}\text { Copernicia prunifera (Mill.) } \\
\text { Moore }\end{array} & \text { H. E. } \\
\end{array}$ & Arecaceae & $\mathrm{U}$ & $\mathrm{A}$ \\
\hline Caroá & Neoglaziovia variegata (Arruda) Mez. & Bromeliaceae & $\mathrm{M} ; \mathrm{U}$ & $\begin{array}{l}\mathrm{E} ; \mathrm{Mm} ; \\
\mathrm{Mp}\end{array}$ \\
\hline Carrapateira & Ricinus communis L. & Euphorbiaceae & $\mathrm{M} ; \mathrm{NU} ; \mathrm{U}$ & $\begin{array}{l}\mathrm{Mg} \\
\mathrm{Mm}\end{array}$ \\
\hline $\begin{array}{l}\text { Carrapicho } \\
\text { de cavalo }\end{array}$ & Cenchrus echinatus L. & Poaceae & L; M; NU; U & $\mathrm{Mp} ; \mathrm{R}$ \\
\hline $\begin{array}{l}\text { Carrapicho } \\
\text { de ovelha }\end{array}$ & Tragus berteronianus Schult. & Poaceae & L; M; NU; U & $\begin{array}{l}\mathrm{C} ; \mathrm{Mp} ; \\
\mathrm{Mr}\end{array}$ \\
\hline Catingueira & $\begin{array}{lll}\text { Cenostigma pyramidale } & \text { (Tul.) } & \text { E. } \\
\text { Gagnon \& G. P. Lewis } & & \\
\end{array}$ & Fabaceae & $\mathrm{M} ; \mathrm{U}$ & $\mathrm{A} ; \mathrm{Mg}$ \\
\hline $\begin{array}{l}\text { Chucalho de } \\
\text { raposa }\end{array}$ & Crotalaria incana L. & Fabaceae & $\mathrm{U}$ & $\mathrm{Mp} ; \mathrm{R}$ \\
\hline $\begin{array}{l}\text { Cidreira } \\
\text { braba }\end{array}$ & Croton muscicarpa Müll. Arg. & Euphorbiaceae & $\mathrm{L} ; \mathrm{U}$ & $\begin{array}{l}\mathrm{Mg} \\
\mathrm{Mm}\end{array}$ \\
\hline $\begin{array}{l}\text { Cidreira } \\
\text { braba }\end{array}$ & Sida cordifolia $\mathrm{L}$. & Malvaceae & $\mathrm{L} ; \mathrm{U}$ & $\begin{array}{l}\mathrm{Mg} \\
\mathrm{Mm}\end{array}$ \\
\hline $\begin{array}{l}\text { Cidreira } \\
\text { braba }\end{array}$ & Lippia microphylla Cham. & Verbenaceae & $\mathrm{L} ; \mathrm{U}$ & $\begin{array}{l}\mathrm{Mg} \\
\mathrm{Mm}\end{array}$ \\
\hline $\begin{array}{l}\text { Cidreira do } \\
\text { mato }\end{array}$ & Lantana camara L. & Verbenaceae & L; M; U & $\begin{array}{l}\mathrm{Mg} \\
\mathrm{Mm}\end{array}$ \\
\hline Cipoeiro & Euphorbia tirucalli L. & Euphorbiaceae & $\mathrm{E}$ & $\mathrm{Mg}$ \\
\hline $\begin{array}{l}\text { Coração de } \\
\text { homem }\end{array}$ & Hibiscus rosa-sinensis L. & Malvaceae & $\mathrm{U}$ & $\mathrm{Mp}$ \\
\hline $\begin{array}{l}\text { Coroa-de- } \\
\text { frade }\end{array}$ & $\begin{array}{l}\text { Melocactus zehntneri (Britton \& } \\
\text { Rose) Luetzelb. }\end{array}$ & Cactaceae & $\mathrm{M} ; \mathrm{U}$ & $\begin{array}{l}\mathrm{E} ; \mathrm{Mp} ; \\
\mathrm{Mr}\end{array}$ \\
\hline $\begin{array}{l}\text { Coronha } \\
\text { braba }\end{array}$ & $\begin{array}{lll}\text { Chloroleucon mangense } & \text { (Jacq.) } \\
\text { Britton \& Rose } & & \\
\end{array}$ & Fabaceae & $\mathrm{M}$ & $\mathrm{Mg}$ \\
\hline Cravo & Tagetes minuta L. & Asteraceae & $\mathrm{L} ; \mathrm{M}$ & $\mathrm{Mp}$ \\
\hline $\begin{array}{l}\text { Cravo-de- } \\
\text { urubu }\end{array}$ & Heliotropium indicum (L.) & Boraginaceae & $\mathrm{L}$ & $\mathrm{Mp}$ \\
\hline
\end{tabular}


Table 1. Continued.

\begin{tabular}{|c|c|c|c|c|}
\hline $\begin{array}{l}\text { Vernacular } \\
\text { name }\end{array}$ & Scientific name & Family & $\begin{array}{l}\text { Classification } \\
\text { criteria }\end{array}$ & $\begin{array}{c}\text { Life } \\
\text { form }\end{array}$ \\
\hline $\begin{array}{l}\text { Crista de } \\
\text { peru; }\end{array}$ & $\begin{array}{l}\text { Heliotropium elongatum (Lehm.) I. M. } \\
\text { Johnst. }\end{array}$ & Boraginaceae & E; M; NU; U & $\begin{array}{l}\mathrm{Mm} ; \\
\mathrm{Mp} ; \mathrm{Mr}\end{array}$ \\
\hline Fedegoso & $\begin{array}{l}\text { Heliotropium elongatum (Lehm.) I. M. } \\
\text { Johnst. }\end{array}$ & Boraginaceae & E; M; NU; U & $\begin{array}{l}\mathrm{Mm} ; \\
\mathrm{Mp} ; \mathrm{Mr}\end{array}$ \\
\hline Cristo reis & Ipomoea sp. & Convolvulaceae & $\mathrm{M} ; \mathrm{U}$ & $\mathrm{Mp}$ \\
\hline Cumaru & $\begin{array}{l}\text { Amburana cearensis (Allemão) A. C. } \\
\text { Sm. }\end{array}$ & Fabaceae & L; M; U & $\mathrm{A} ; \mathrm{Mg}$ \\
\hline Cumbeba & $\begin{array}{l}\text { Tacinga inamoena (K. Schum.) N. P. } \\
\text { Taylor \& Stuppy }\end{array}$ & Cacataceae & $\mathrm{M} ; \mathrm{U}$ & $\begin{array}{l}\text { E; Mp; } \\
\mathrm{Mr}\end{array}$ \\
\hline Endro & Foeniculum vulgare Gaertn. & Apiaceae & $\mathrm{M} ; \mathrm{U}$ & $\mathrm{Mp}$ \\
\hline $\begin{array}{l}\text { Espinho de } \\
\text { cigano }\end{array}$ & Acanthospermum hispidum DC. & Asteraceae & L; M; NU; U & Mp; R \\
\hline $\begin{array}{l}\text { Esterco de } \\
\text { passarinho }\end{array}$ & $\begin{array}{l}\text { Phoradendron mucronatum (DC.) } \\
\text { Krug \& Urb. }\end{array}$ & Viscaceae & $\mathrm{U}, \mathrm{E}$ & $\mathrm{R} ; \mathrm{Mr}$ \\
\hline Eva babosa & Aloe vera (L.) Burm.f. & Liliaceae & $\mathrm{M} ; \mathrm{U}$ & $\begin{array}{ll}\text { A; } & \text { E; } \\
\mathrm{Mp} & \end{array}$ \\
\hline Eva cidreira & Lippia alba (Mill.) N. E. Br. & Verbenaceae & $\mathrm{M} ; \mathrm{U}$ & $\begin{array}{l}\mathrm{Mm} ; \\
\mathrm{Mp}\end{array}$ \\
\hline Eva doce & Pimpinella anisum L. & Apiaceae & L; M; U & $\mathrm{Mp}$ \\
\hline Facheiro & $\begin{array}{l}\text { Pilosocereus pachycladus F. Ritter } \\
\text { subsp. pernambucoensis (F. Ritter) } \\
\text { Zappi }\end{array}$ & Cactaceae & $\mathrm{M} ; \mathrm{U}$ & $\mathrm{E} ; \mathrm{Mg}$ \\
\hline Favela & Cnidoscolus quercifolius Pohl & Euphorbiaceae & $\mathrm{M} ; \mathrm{U}$ & $\begin{array}{l}\mathrm{A} ; \mathrm{Mg} ; \\
\mathrm{Mm}\end{array}$ \\
\hline $\begin{array}{l}\text { Favela } \\
\text { branca }\end{array}$ & Cnidoscolus quercifolius Pohl & Euphorbiaceae & $\mathrm{M} ; \mathrm{U}$ & $\mathrm{A} ; \mathrm{Mg}$ \\
\hline Feijão brabo & Cynophalla flexuosa (L.) J. Presl & Capparaceae & $\mathrm{M} ; \mathrm{U}$ & $\mathrm{A} ; \mathrm{Mg}$ \\
\hline $\begin{array}{l}\text { Feijão de } \\
\text { rolinha }\end{array}$ & Desmanthus virgatus (L.) Willd. & Fabaceae & $\mathrm{M} ; \mathrm{U}$ & Mr; R \\
\hline Girassol & Helianthus annuus & Asteraceae & $\mathrm{U}$ & $\mathrm{Mp}$ \\
\hline Godião & Secondatia cf. densiflora A. DC & Apocynaceae & $\mathrm{M} ; \mathrm{U}$ & $\mathrm{Mp}$ \\
\hline Gogoia & Solanum agrarium Sendtn & Solanaceae & $\mathrm{M} ; \mathrm{NU}$ & $\mathrm{E} ; \mathrm{Mp}$ \\
\hline $\begin{array}{l}\text { Hortelã do } \\
\text { Pará }\end{array}$ & Plectranthus barbatus Andr. & Lamiaceae & L; M; U & $\begin{array}{l}\mathrm{Mm} \\
\mathrm{Mp}\end{array}$ \\
\hline $\begin{array}{l}\text { Hortelã } \\
\text { Graúdo }\end{array}$ & $\begin{array}{l}\text { Plectranthus amboinicus (Lour.) } \\
\text { Spreng. }\end{array}$ & Lamiaceae & L; M; U & $\begin{array}{l}\mathrm{Mm} ; \\
\mathrm{Mp}\end{array}$ \\
\hline $\begin{array}{l}\text { Hortelã } \\
\text { miúdo }\end{array}$ & Mentha piperita L. & Lamiaceae & L; M; U & $\begin{array}{l}\mathrm{Mm} ; \\
\mathrm{Mp}\end{array}$ \\
\hline Icó & $\begin{array}{l}\text { Neocalyptrocalyx longifolium (Mart.) } \\
\text { Cornejo \& Iltis }\end{array}$ & Capparaceae & M & $\mathrm{Mg}$ \\
\hline Imbiratã & $\begin{array}{l}\text { Pseudobombax marginatum (A. } \\
\text { St.-Hil., Juss. \& Cambess.) A. Robyns }\end{array}$ & Bombacaceae & $\mathrm{M} ; \mathrm{U}$ & $\mathrm{A} ; \mathrm{Mg}$ \\
\hline Ingazeira & Inga sp. & Fabaceae & $\mathrm{M} ; \mathrm{U}$ & $\mathrm{A} ; \mathrm{Mg}$ \\
\hline Jaboticabra & Myrciaria sp. & Myrtaceae & $\mathrm{M} ; \mathrm{U}$ & $\mathrm{Mg}$ \\
\hline Jatobá & Hymenaea sp. & Fabaceae & $\mathrm{M} ; \mathrm{U}$ & $\mathrm{Mg}$ \\
\hline João mole & Thiloa glaucocarpa (Mart.) Eichler & Combretaceae & $\mathrm{M} ; \mathrm{U}$ & $\mathrm{A} ; \mathrm{Mg}$ \\
\hline Juazeiro & Ziziphus joazeiro Mart. & Rhamnaceae & $\mathrm{M} ; \mathrm{U}$ & $\mathrm{A} ; \mathrm{Mg}$ \\
\hline Jucá & $\begin{array}{l}\text { Libidibia ferrea (Mart. ex Tul.) L. P. } \\
\text { Queiroz }\end{array}$ & Fabaceae & $\mathrm{M} ; \mathrm{U}$ & $\begin{array}{l}\mathrm{A} ; \mathrm{Mg} ; \\
\mathrm{Mm}\end{array}$ \\
\hline
\end{tabular}


Table 1. Continued.

\begin{tabular}{|c|c|c|c|c|}
\hline $\begin{array}{l}\text { Vernacular } \\
\text { name }\end{array}$ & Scientific name & Family & $\begin{array}{c}\text { Classification } \\
\text { criteria }\end{array}$ & $\begin{array}{l}\text { Life } \\
\text { form }\end{array}$ \\
\hline Jucuri & Pithecelobium diversifolium Benth. & Fabaceae & $\mathrm{S}$ & $\mathrm{Mg}$ \\
\hline $\begin{array}{l}\text { Jurema } \\
\text { branca }\end{array}$ & $\begin{array}{l}\text { Piptadenia stipulaceae } \\
\text { Ducke }\end{array}$ & Fabaceae & L; M; NU; U & $\mathrm{A} ; \mathrm{Mg}$ \\
\hline $\begin{array}{l}\text { Jurema de } \\
\text { imbira }\end{array}$ & $\begin{array}{l}\text { Mimosa ophthalmocentra Mart ex } \\
\text { Benth. }\end{array}$ & Fabaceae & L; M; U & $\begin{array}{l}\mathrm{A} ; \mathrm{Mg} ; \\
\mathrm{Mm}\end{array}$ \\
\hline $\begin{array}{l}\text { Jurema } \\
\text { preta }\end{array}$ & Mimosa tenuiflora (Willd.) Poir. & Fabaceae & L; M; U & $\begin{array}{l}\mathrm{A} ; \mathrm{Mg} ; \\
\mathrm{Mm}\end{array}$ \\
\hline Louro & $\begin{array}{l}\text { Cordia trichotoma (Vell.) Arrab. ex } \\
\text { Steud. }\end{array}$ & Boraginaceae & $\mathrm{U}$ & $\mathrm{Mg}$ \\
\hline $\begin{array}{l}\text { Macambira } \\
\text { branca }\end{array}$ & $\begin{array}{l}\text { Encholirium spectabile } \\
\text { Schult. \& Schult. f. }\end{array}$ & Bromeliaceae & $\mathrm{U}$ & $\mathrm{E} ; \mathrm{Mp}$ \\
\hline $\begin{array}{l}\text { Macambira } \\
\text { de pedra }\end{array}$ & Encholirium sp. & Bromeliaceae & L; M; U & $\mathrm{E} ; \mathrm{Mp}$ \\
\hline $\begin{array}{l}\text { Macambira } \\
\text { roxa }\end{array}$ & Bromelia laciniosa Mart. ex Schult. f. & Bromeliaceae & L; M; U & $\mathrm{E} ; \mathrm{Mp}$ \\
\hline Macela & Egletes viscosa (L.) Less. & Asteraceae & $\mathrm{U}$ & $\mathrm{Mp}$ \\
\hline Malicia & Chamaecrista rotundefolia Greene & Fabaceae & $\mathrm{M} ; \mathrm{NU}$ & $\begin{array}{l}\mathrm{Mm} ; \\
\mathrm{Mp}\end{array}$ \\
\hline Malva & Sida galheirensis Ulbr. & Malvaceae & $\mathrm{M} ; \mathrm{NU} ; \mathrm{U}$ & $\mathrm{Mp}$ \\
\hline $\begin{array}{l}\text { Malva } \\
\text { branca }\end{array}$ & Waltheria rotundifolia Schrank & Malvaceae & $\mathrm{M} ; \mathrm{NU} ; \mathrm{U}$ & $\mathrm{Mp}$ \\
\hline Mamona & Ricinus communis L. & Euphorbiaceae & $\mathrm{M}$ & $\mathrm{Mm}$ \\
\hline Malva rosa & Melochia tomentosa $\mathrm{L}$. & Sterculiaceae & $\mathrm{M} ; \mathrm{Nu} ; \mathrm{U}$ & $\mathrm{Mp}$ \\
\hline Maniçoba & Manihot dichotomaUle & Euphorbiaceae & $\mathrm{M} ; \mathrm{NU} ; \mathrm{U}$ & $\mathrm{A} ; \mathrm{Mg}$ \\
\hline Manjericão & Ocimum basilicum L. & Lamiaceae & $\mathrm{L} ; \mathrm{M} ; \mathrm{U}$ & $\mathrm{Mp}$ \\
\hline Manjirioba & Senna occidentalis (L.) Link. & Fabaceae & $\mathrm{L} ; \mathrm{M} ; \mathrm{U}$ & $\mathrm{Mp}$ \\
\hline Mão fechada & Selaginella convoluta (Arn.) Spring. & Selaginellaceae & $\mathrm{M} ; \mathrm{U}$ & $\mathrm{Mp} ; \mathrm{Mr}$ \\
\hline $\begin{array}{l}\text { Maracujá do } \\
\text { mato }\end{array}$ & Passiflora foetida $\mathrm{L}$. & Passifloraceae & E; M; U & $\mathrm{Mp} ; \mathrm{R}$ \\
\hline $\begin{array}{l}\text { Maria de } \\
\text { três } \\
\text { babados }\end{array}$ & Hyptis sp. & Lamiaceae & $\mathrm{M} ; \mathrm{U}$ & $\mathrm{Mp}$ \\
\hline Maria preta & Cordia leucocephala Moric. & Boraginaceae & $\mathrm{M}$ & $\mathrm{Mg}$ \\
\hline Marmeleiro & Croton blanchetianus Baill. & Euphorbiaceae & L; M; U & $\begin{array}{l}\mathrm{Mg} \\
\mathrm{Mm}\end{array}$ \\
\hline $\begin{array}{l}\text { Marmeleiro } \\
\text { branco }\end{array}$ & Croton sincorensis Mart. & Euphorbiaceae & L; M; U & $\mathrm{Mm}$ \\
\hline Mastruz & Chenopodium ambrosioides L. & Chenopodiaceae & $\mathrm{M} ; \mathrm{U}$ & $\mathrm{Mp}$ \\
\hline Mata fome & Serjania glabrata Kunth. & Sapindaceae & M; NU; U & $\begin{array}{l}\text { C; } \mathrm{Mp} ; \\
\text { Mr; R }\end{array}$ \\
\hline Mata pasto & $\begin{array}{l}\text { Senna obtusifolia (L.) H. S. Irwin \& } \\
\text { Barneby }\end{array}$ & Fabaceae & $\mathrm{M} ; \mathrm{NU} ; \mathrm{U}$ & $\begin{array}{l}\mathrm{C} ; \mathrm{Mm} ; \\
\mathrm{Mp}\end{array}$ \\
\hline Mato azul & Heliotropium procumbens Mill. & Boraginaceae & $\mathrm{M} ; \mathrm{U}$ & $\begin{array}{l}\mathrm{C} ; \mathrm{Mm} ; \\
\mathrm{Mp}\end{array}$ \\
\hline $\begin{array}{l}\text { Maxixe } \\
\text { brabo }\end{array}$ & Cucumus anguria L. & Cucurbitaceae & $\mathrm{L}$ & $\mathrm{Mp}$ \\
\hline Mela bode & Herissantia crispa (L.) Brizicky & Malvaceae & $\mathrm{M} ; \mathrm{U}$ & $\begin{array}{l}\text { Mm; } \\
\mathrm{Mp} ; \mathrm{Mr}\end{array}$ \\
\hline Meladinha & Stemodia marítima L. & Scrophulariaceae & $\mathrm{M}$ & $\mathrm{Mm}$ \\
\hline $\begin{array}{l}\text { Melancia de } \\
\text { vaca }\end{array}$ & $\begin{array}{l}\text { Richardia grandiflora (Cham. \& } \\
\text { Schltdl.) Steud. }\end{array}$ & Rubiaceae & $\mathrm{M} ; \mathrm{U}$ & $\begin{array}{l}\text { Mp; } \\
\text { Mr; R }\end{array}$ \\
\hline
\end{tabular}


Table 1. Continued.

\begin{tabular}{|c|c|c|c|c|}
\hline $\begin{array}{l}\text { Vernacular } \\
\text { name }\end{array}$ & Scientific name & Family & $\begin{array}{c}\text { Classification } \\
\text { criteria }\end{array}$ & $\begin{array}{c}\text { Life } \\
\text { form }\end{array}$ \\
\hline $\begin{array}{l}\text { Melão de } \\
\text { São Caetano }\end{array}$ & Momordica charantia L. & Cucurbitaceae & $\mathrm{M} ; \mathrm{U}$ & $\begin{array}{l}\mathrm{Mp} ; \\
\mathrm{Mr} ; \mathrm{R}\end{array}$ \\
\hline Mororó & Bauhinia cheilantha (Bong.) Steud. & Fabaceae & $\mathrm{M} ; \mathrm{U}$ & $\mathrm{A} ; \mathrm{Mg}$ \\
\hline Mufumbu & Combretum fruticosum (Loefl.) Stuntz & Combretaceae & L; M; U & $\begin{array}{l}\mathrm{C} ; \mathrm{Mg} ; \\
\mathrm{Mm}\end{array}$ \\
\hline Mulungu & Erythrina velutina Willd. & Fabaceae & $\mathrm{M} ; \mathrm{U}$ & $\mathrm{A} ; \mathrm{Mg}$ \\
\hline Mussambê & Tarenaya spinosa (Jacq.) Raf & Cleomaceae & E; M; NU; U & $\begin{array}{l}\mathrm{Mm}, \\
\mathrm{Mp}\end{array}$ \\
\hline Neuvagina & $\begin{array}{l}\text { Alternanthera dentata (Moench.) } \\
\text { Stuchlik. ex. R.E. Fr. }\end{array}$ & Amaranthaceae & $\mathrm{S}$ & $\mathrm{Mp}$ \\
\hline Oliveira & Nicotiana glauca R. Grah. & Solanaceae & $\mathrm{M} ; \mathrm{NU}$ & $\mathrm{Mg}$ \\
\hline Palma & Opuntia ficus-indica (L.) Mill. & Cactaceae & $\mathrm{M} ; \mathrm{U}$ & $\mathrm{E} ; \mathrm{Mg}$ \\
\hline Palmatória & $\begin{array}{l}\text { Tacinga palmadora (Britton \& Rose) } \\
\text { N. P. Taylor \& Stuppy }\end{array}$ & Cactaceae & $\mathrm{M} ; \mathrm{U}$ & $\mathrm{E} ; \mathrm{Mg}$ \\
\hline Pega pinto & Boerhavia diffusa L. & Nyctaginaceae & $\mathrm{M} ; \mathrm{U}$ & $\mathrm{Mp} ; \mathrm{Mr}$ \\
\hline Penicilina & Alternanthera sp. & Amaranthaceae & $\mathrm{U}$ & $\mathrm{Mp}$ \\
\hline Pereiro & $\begin{array}{l}\text { Aspidosperma pyrifolium Mart. \& } \\
\text { Zucc. }\end{array}$ & Apocynaceae & $\mathrm{M} ; \mathrm{U}$ & $\mathrm{A} ; \mathrm{Mg}$ \\
\hline $\begin{array}{l}\text { Pimenta } \\
\text { dágua }\end{array}$ & Calliandra sp. & Fabaceae & L; M; U & $\mathrm{C} ; \mathrm{Mm}$ \\
\hline $\begin{array}{l}\text { Pinhão } \\
\text { brabo }\end{array}$ & Jatropha mollissima (Pohl) Baill. & Euphorbiaceae & L; M; NU & $\begin{array}{l}\mathrm{Mg} ; \\
\mathrm{Mm}\end{array}$ \\
\hline $\begin{array}{l}\text { Pinhão } \\
\text { manso }\end{array}$ & Jatropha ribifolia (Pohl) Baill. & Euphorbiaceae & L; M; NU & $\begin{array}{l}\mathrm{Mg} ; \\
\mathrm{Mm}\end{array}$ \\
\hline Pinhão roxo & Jatrophagos sypiifolia L. & Euphorbiaceae & L; M; MR; NU & $\begin{array}{l}\mathrm{Mg} ; \\
\mathrm{Mm}\end{array}$ \\
\hline Pratude & Mollugo verticillata $\mathrm{L}$. & Sapindaceae & $\mathrm{M}$ & $\mathrm{C} ; \mathrm{Mp}$ \\
\hline Pratude & Cardiospermum corindum L. & Sapindaceae & $\mathrm{M}$ & $\mathrm{C} ; \mathrm{Mp}$ \\
\hline Quebra faca & Croton heliotropiifolius Kunth. & Euphorbiaceae & $\mathrm{M} ; \mathrm{U}$ & $\begin{array}{l}\mathrm{Mg} \\
\mathrm{Mm}\end{array}$ \\
\hline $\begin{array}{l}\text { Quebra } \\
\text { panela }\end{array}$ & Alternanthera tenella Colla & Amaranthaceae & $\mathrm{M} ; \mathrm{U}$ & $\begin{array}{l}\mathrm{Mm} ; \\
\mathrm{Mp}\end{array}$ \\
\hline $\begin{array}{l}\text { Quebra } \\
\text { pedra }\end{array}$ & Phyllanthus sp. & Euphorbiaceae & $\mathrm{M} ; \mathrm{U}$ & $\mathrm{Mp} ; \mathrm{Mr}$ \\
\hline Quixabeira & $\begin{array}{l}\text { Sideroxylon obtusifolium (Roem. \& } \\
\text { Schult.) T. D. Penn. }\end{array}$ & Sapotaceae & $\mathrm{M} ; \mathrm{U}$ & $\mathrm{A} ; \mathrm{Mg}$ \\
\hline $\begin{array}{l}\text { Quixabeira } \\
\text { branca }\end{array}$ & $\begin{array}{l}\text { Sideroxylon obtusifolium (Roem. \& } \\
\text { Schult.) T. D. Penn. }\end{array}$ & Sapotaceae & $\mathrm{M} ; \mathrm{U}$ & $\mathrm{A} ; \mathrm{Mg}$ \\
\hline Romã & Punica granatum & Lythraceae & $\mathrm{U}$ & $\mathrm{Mp}$ \\
\hline $\begin{array}{ll}\text { Sabão } & \text { de } \\
\text { soldado } & \\
\end{array}$ & Microtea paniculata Moq. & Phytolaccaceae & $\mathrm{U}$ & $\mathrm{Mp}$ \\
\hline Saião & Kalanchoe brasilensis Camb. & Crassulaceae & $\mathrm{M} ; \mathrm{U}$ & $\mathrm{Mp}$ \\
\hline Salambaia & $\begin{array}{l}\text { Tillandsia bulbosa Hook. } \\
\text { Tillandsia recurvata (L.) L. }\end{array}$ & Bromeliaceae & E; M; NU; U & $\begin{array}{l}\mathrm{Mm} ; \\
\mathrm{Mp} ; \mathrm{R}\end{array}$ \\
\hline $\begin{array}{l}\text { Salambaia } \\
\text { do pinhão } \\
\text { brabo }\end{array}$ & Tillandsia streptocarpa Baker & Bromeliaceae & $\mathrm{U}$ & $\mathrm{C}$ \\
\hline Tamiarana & Tragia volubilis L. & Euphorbiaceae & $\mathrm{M} ; \mathrm{NU}$ & $\mathrm{Mp}$ \\
\hline Turco & Parkinsonia aculeata L. & Fabaceae & $\mathrm{M} ; \mathrm{U}$ & $\mathrm{Mg}$ \\
\hline Ubaia & Eugenia pyriformis Cambess. & Myrtaceae & $\mathrm{M}$ & $\mathrm{A} ; \mathrm{Mg}$ \\
\hline
\end{tabular}


Table 1. Continued.

\begin{tabular}{|l|l|l|l|l|}
\hline $\begin{array}{l}\text { Vernacular } \\
\text { name }\end{array}$ & \multicolumn{1}{|c|}{ Scientific name } & \multicolumn{1}{|c|}{ Family } & $\begin{array}{c}\text { Classification } \\
\text { criteria }\end{array}$ & $\begin{array}{c}\text { Life } \\
\text { form }\end{array}$ \\
\hline Umburana & $\begin{array}{l}\text { Commiphora leptophloeos (Mart.) J. B. } \\
\text { Gillet. }\end{array}$ & Burseraceae & $\mathrm{M} ; \mathrm{U}$ & $\mathrm{A} ; \mathrm{Mg}$ \\
\hline Umbuzeiro & Spondias tuberosa Arruda & Anacardiaceae & $\mathrm{M} ; \mathrm{U}$ & $\mathrm{A} ; \mathrm{Mg}$ \\
\hline Urtiga & $\begin{array}{l}\text { Cnidoscolus loefgrenii (Pax \& K. } \\
\text { Hoffm.) Pax \& K. Hoffm. }\end{array}$ & Euphorbiaceae & $\mathrm{M} ; \mathrm{NU}$ & $\mathrm{Mp}$ \\
\hline $\begin{array}{l}\text { Urtiga } \\
\text { branca }\end{array}$ & Aosa rupestris (Gardner) Weigend & Loasaceae & $\mathrm{M} ; \mathrm{U}$ & $\mathrm{Mp}$ \\
\hline Vá moura & Aureliana fasciculata (Vell.) Sendtn. & Solanaceae & $\mathrm{M} ; \mathrm{NU}$ & $\mathrm{Mg}$ \\
\hline Velame & Crotonheliotropii folius Kunth. & Euphorbiaceae & $\mathrm{M} ; \mathrm{U}$ & $\mathrm{Mg}$ \\
\hline Xiquexique & $\begin{array}{l}\text { Pilosocereus gounellei (F. A. C. } \\
\text { Weber) Byles \& Rowley }\end{array}$ & Cactaceae & $\mathrm{M} ; \mathrm{U}$ & $\mathrm{E} ; \mathrm{Mm}$ \\
\hline Zazumba & Leonotisnepetifolia (L.) R.Br. & Lamiaceae & $\mathrm{S}$ & $\mathrm{Mp}$ \\
\hline
\end{tabular}

The local specialists proved to have a wide knowledge of plant diversity in their communities; however, none of them were able to identify all plant species cited in the interviews, despite most of them belonging to an ordinary domain. On average, $60.5 \%$ of the folk generics mentioned in the general interviews were identified in Cachoeira, but three specialists individually identified more than $90 \%$ of the 201 folk generics. Barrocas accounted for an average of $43 \%$, and one specialist identified $97 \%$ and another $85.5 \%$ of folk generics. All woody species were identified by local specialists. There were distinctions regarding the small herbaceous, such as in the case of species belonging to families Poaceae and Lamiaceae. This knowledge contributed to elaborating a representative taxonomy of the studied human populations.

The criteria used by the local specialists to classify and group the plants were based on utilitarian, morphological, ecological, linguistic, and non-utilitarian aspects. In Cachoeira, morphological criteria were used by $100 \%$ of the local specialists, utilitarian by $83 \%$, linguistic and non-utilitarian by $67 \%$, and ecological by $42 \%$. In Barrocas, $80 \%$ of them used morphological and utilitarian criteria (although they have not necessarily been used by the same people), $60 \%$ used non-utilitarian and 20\% linguistic criteria. In addition to these groups, some folk generics were isolated because they shared no characteristics which could include them in the abovementioned groups. Such groups were formed as follow: (1) "jurema branca", "jurema preta", and "jurema de imbira" because they have similar leaves and stems (morphological criterion) (Figure 3); (2) "pinhão brabo" and "pinhão manso" because they have the same name "de boi" and because they grow on other plants (ecological); (5) "vá moura" and "urtiga" for having no utility to people or animals (non-utilitarian); and (6) "língua de vaca", "agulha braba", and "pinhão roxo" classified as isolated generics because they shared no characteristics with other plants. "pinhão" (linguistic) (Figure 4); (3) "hortelã miúda", "hortelã graúda", and "lemongrass", used for medicinal purpose (utilitarian) (Figure 5). 


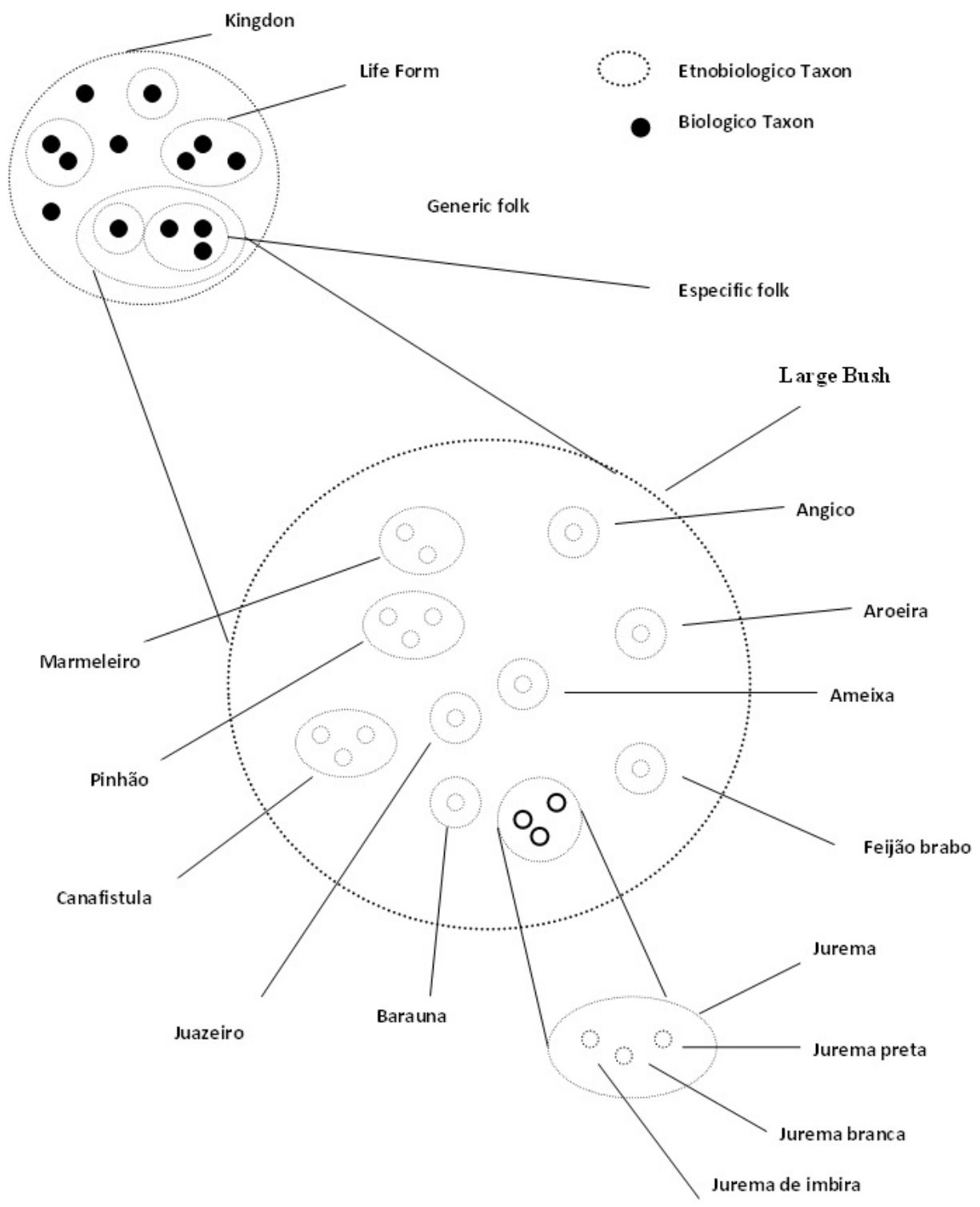

Figure 2. Schematic representation of the four classification categories and their ethnobiological taxa in concurrent use with the hierarchical classification in rural communities of Soledade (Paraíba, Northeastern Brazil), following the model proposed by Berlin (1992).

Forty-five percent of the folk generics were classified based on two criteria, and $28 \%, 23 \%$, and $4 \%$ used one, three, and four criteria, respectively. These results were obtained by considering all classifications together of all specialists in Barrocas and Cachoeira. The fact that a folk generic has been identified based on more than one criterion is because larger groups were divided into sub-groups and because all the abovementioned information was considered together. Thus, Cactaceae and bromeliad species were grouped in the same group, as they are used as animal feed; however, the division into sub-groups adopted morphological criteria such as leaf type, thorn shape, and plant height. The criteria choice used to define the larger groups and sub-groups varied among the informants and within the classification made by the same specialist. One moment morphological criterion was chosen as the general guide for classification, using the others 
as secondary criteria, the next the utilitarian criterion was chosen as the general guide, and the others were used as secondary criteria.

From these groupings, different species and botanical families shared the same group, such as the generics "capim panasco" (Poaceae) and "barba de bode" (Cyperaceae) because they are animal feed, accounting for $40 \%$ presence in the classification performed in Barrocas and 42\% in Cachoeira, and "caroá" (Bromeliaceae) and "agave" (Agavaceae), which are used to produce handmade ropes, which had $20 \%$ and $25 \%$ presence in the classifications made in Barrocas and Cachoeira, respectively. There were cases in which species belonging to the same family were classified in the same group, similar to scientific classification, such as "hortelã miúda" and "hortelã graúda", which belong to the family Lamiaceae (morphological and utilitarian criteria - medicinal), with $100 \%$ presence in the classification made in Barrocas and 75\% in Cachoeira, and "baraúna" and "aroeira" of the family Anacardiaceae (morphological criterion - leaves, wood, and height similarities) with 100\% presence in the classification performed in Barrocas and 75\% in Cachoeira.

Twelve life forms were recorded, as follows: trees, grass, liana, thorn, large bush, medium bush, small bush, squat bush, underground bush, forage cactus, parasite, and foliage. The life form "tree" was identified by $50 \%$ of the informants in Cachoeira and $40 \%$ in Barrocas; grass by $17 \%$ only in Cachoeira; liana by $50 \%$ in Cachoeira and $20 \%$ in Barrocas; thorn by $83 \%$ in Cachoeira and $60 \%$ in Barrocas; large bush by $83 \%$ in Cachoeira and $100 \%$ in Barrocas; medium bush by $75 \%$ in Cachoeira and $100 \%$ in Barrocas; small bush by $100 \%$ in both communities; squat bush by $16 \%$ in Cachoeira and $20 \%$ in Barrocas; underground bush by $20 \%$ in Barrocas; forage cactus by $8 \%$ in Cachoeira; parasite by $20 \%$ in Barrocas; and foliage by $33 \%$ in Cachoeira and $40 \%$ in Barrocas. From a quantitative point of view, these data indicated large bush, medium bush, small bush, and thorn as the main life forms in both communities.

The results from taxonomic correspondences were obtained by the analysis of 136 species of which collected specimens were identified, indicating 127 correspondences 1:1, four over-differentiation and, one sub-differentiation No. 1.

The pile sort analysis showed no consensus classification system among specialists in the community of Cachoeira because there was no distinction of specific groups (Figure 6). Moreover, the specialists had distinct positions and were separated during the analysis (Figure 7). Even in this context, there was a tendency to group the species following the principles related to the life forms, highlighting a group of woody plants, another of herbaceous, and the third group of cacti and bromeliads, which was classified by the specialists as "thorn" (Figure 8). The other species, which were represented by lianas, epiphytes, and tuberous plants, one moment they were bordering these groups, the next were overlapping them.

In Barrocas, there was a strong tendency to group the species based on morphological and utilitarian characteristics (Figure 9a,b), resulting in groups of plants used for veterinary, medicinal, fodder, and ornamental (utilitarian criteria) purposes, and groups including the folk generics "cabeça de nêgo" and "batata de purga", and "salambaia" and "esterco de passarinho" (morphological criteria). Woody plants were grouped in one group, and cacti, bromeliads, creepers, tuberous plants, and herbaceous were distributed into small groups according to their utilitarian characteristics. The analysis of the species selected based on a conservation point of view in Barrocas showed two distinct groups. The first was formed by $M$. urundeuva and $S$. brasiliensis and the second one comprised species in the marginal zone of the large group of woody species, e.g., C. leptophloeos, A. pyrifolium, $X$. americana, and S. obtusifolium. P. pyramidalis remained apart from the other species, and $C$. blanchetianus was the only one next to the general group center (Figure 10). The woody species identified in Cachoeira remained located and dispersed in a long strip, without forming a larger and more concentrated group. The species selected for observation showed different behavior in Cachoeira in comparison with those 
recorded in Barrocas, because two groups were formed in this latter community: one composed of $S$. brasiliensis, X. americana, and S. obtusifolium, and another including C. blanchetianus, M. urundeuva, A. pyrifolium, and P. pyramidalis. C. leptophloeos shared no group with the other species, appearing isolated (alone) in the analysis.

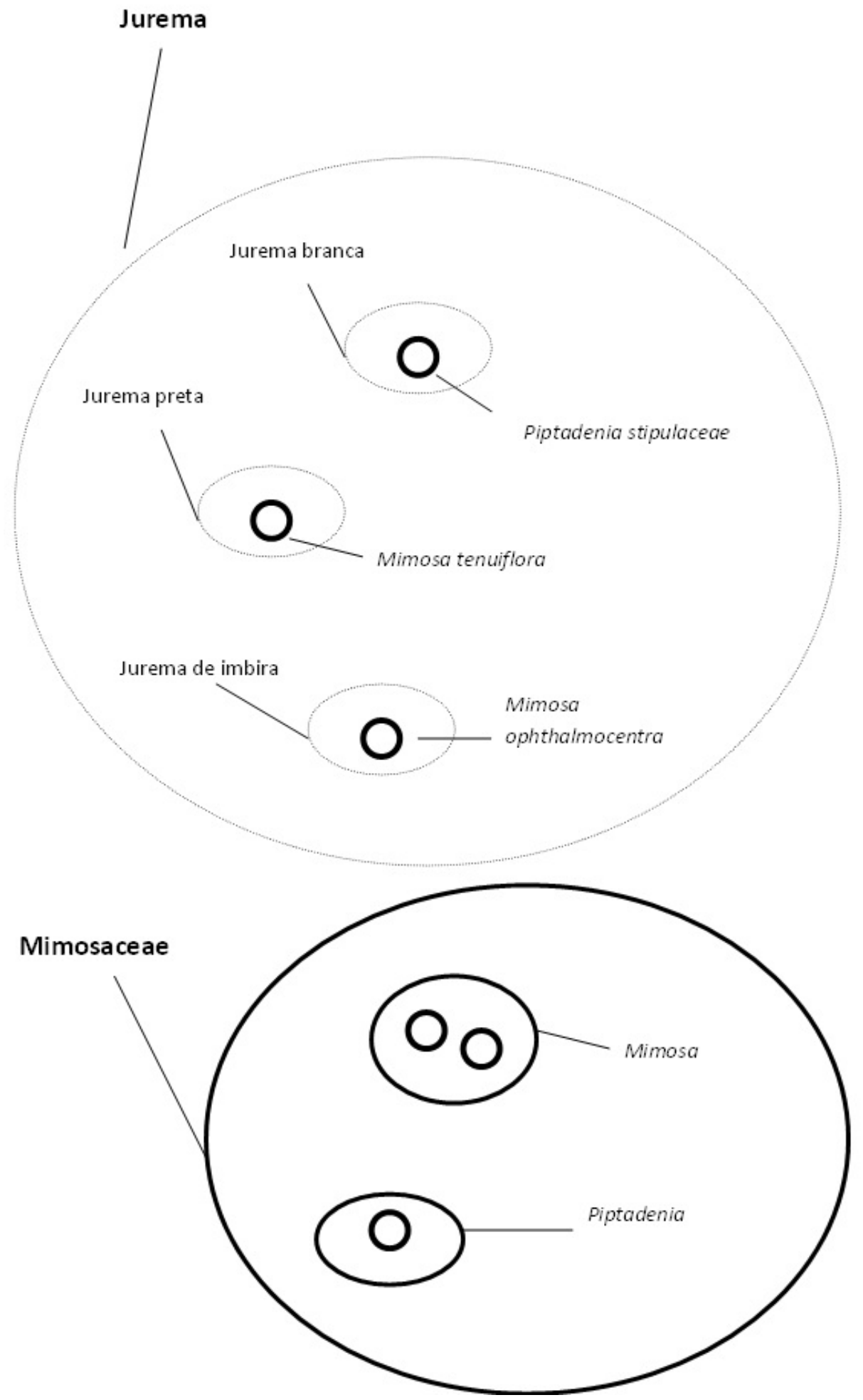

Figure 3. Specific folk generic "Jurema", cited by local specialists in rural communities of Soledade (Paraíba, Northeastern Brazil), and its equivalent names in scientific classification. 

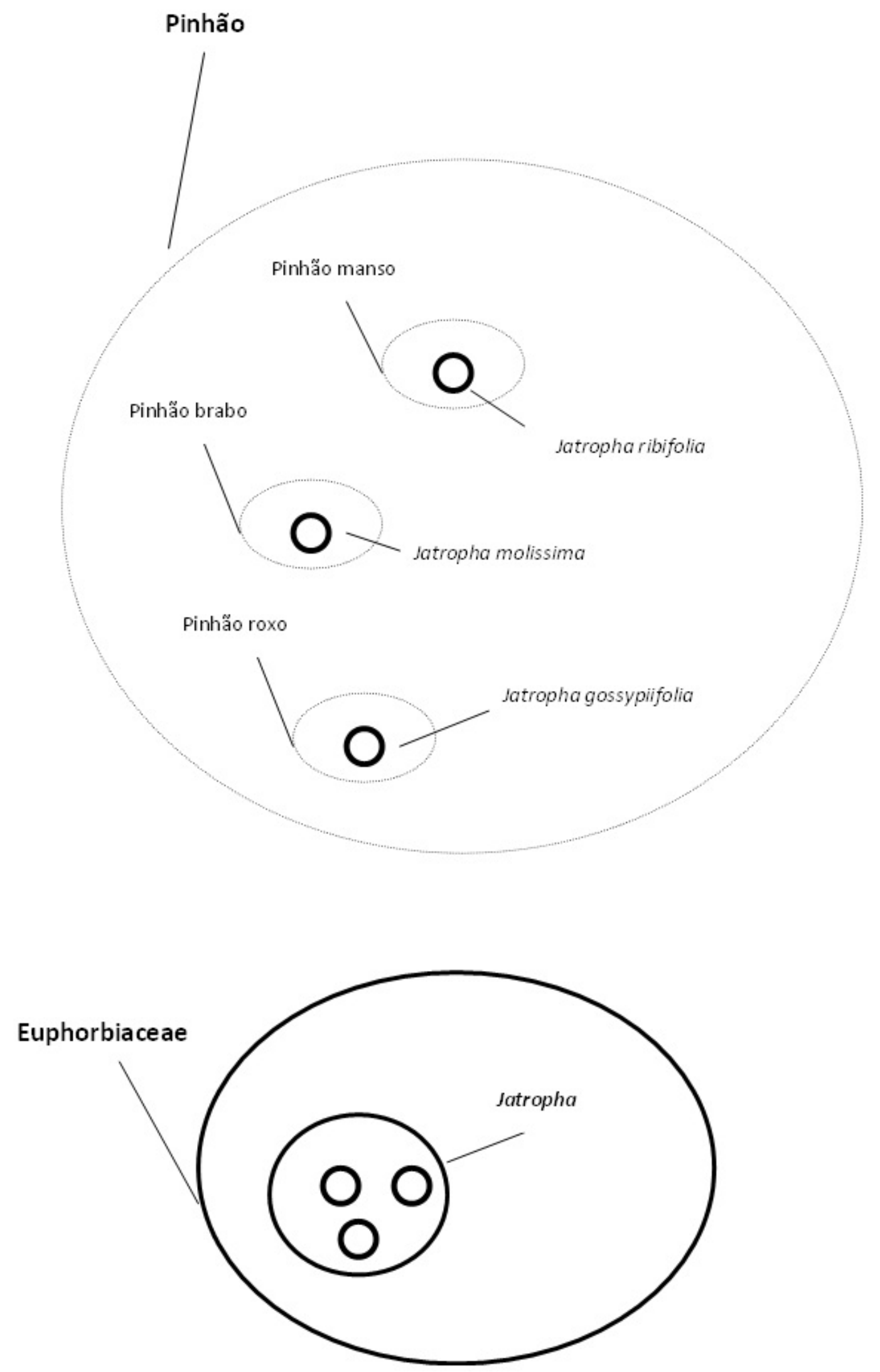

Figure 4. Specific folk generic "Pinhão", cited by local specialists in rural communities of Soledade (Paraíba, Northeastern Brazil), and its equivalent names in scientific classification. 


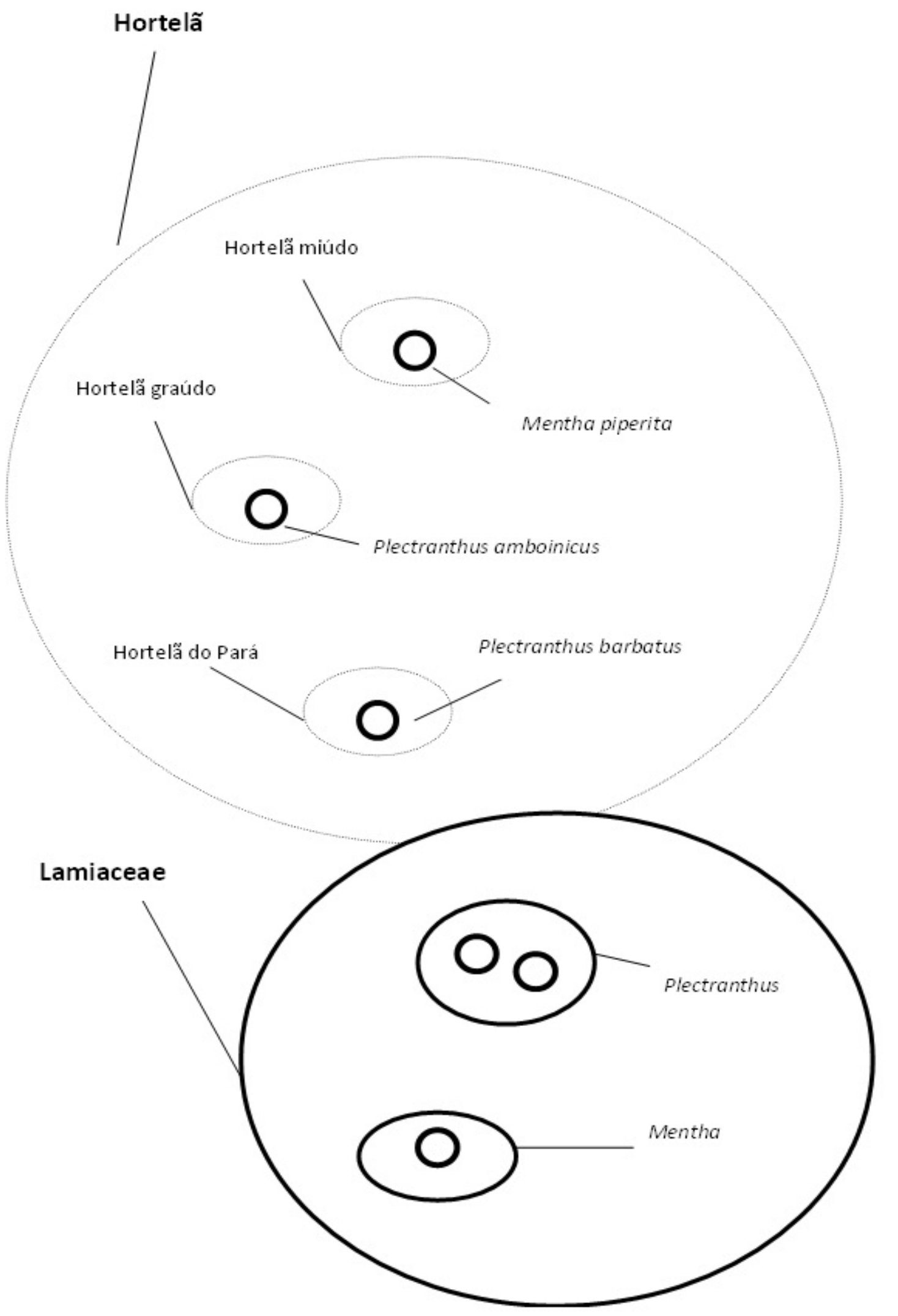

Figure 5. Specific folk generic "Hortelã", cited by local specialists in rural communities of Soledade (Paraíba, Northeastern Brazil), and its equivalent names in scientific classification. 


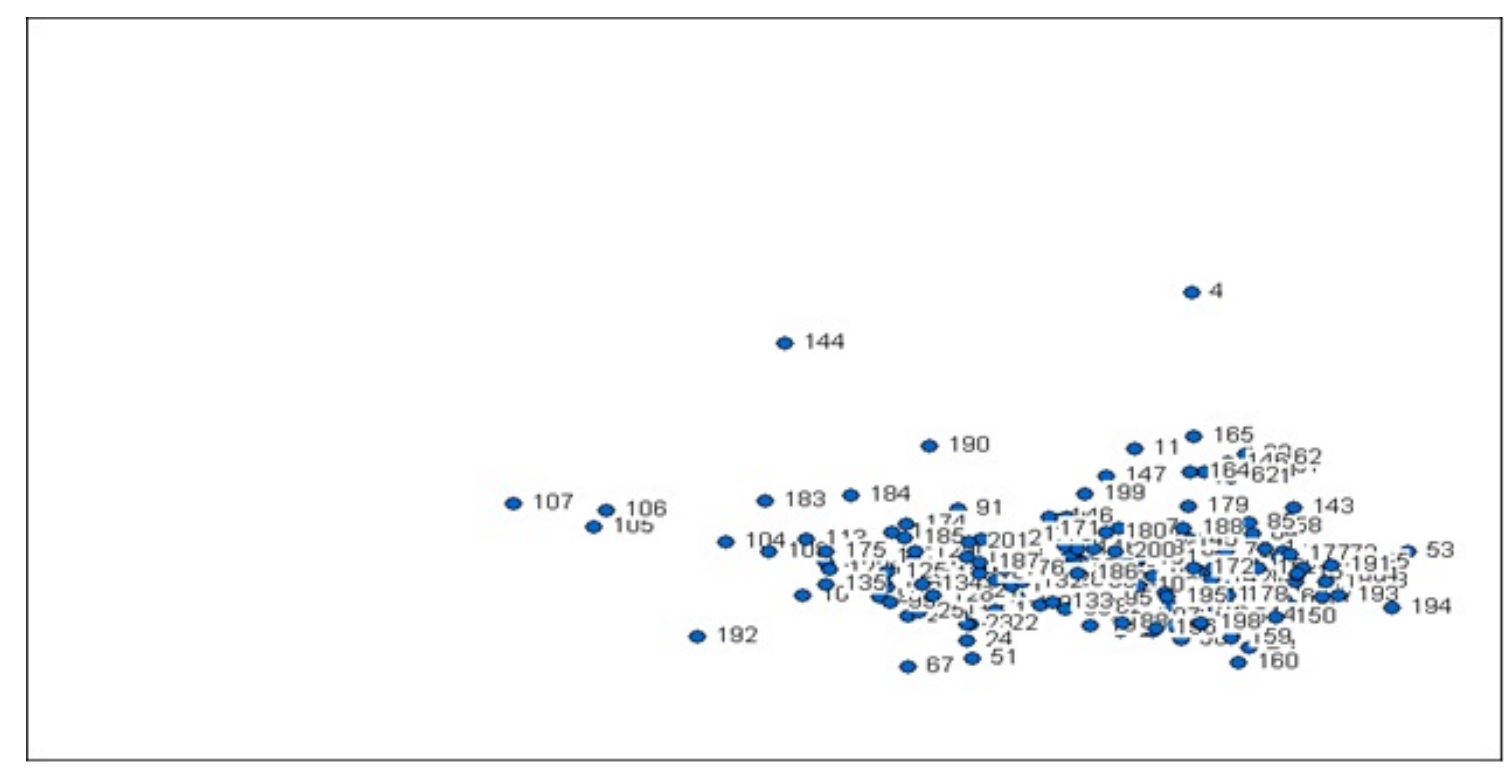

Figure 6. Grouping of folk generics, cited by local specialists in the community of Cachoeira, located in the Municipality of Soledade (Paraíba, Northeastern Brazil), using the pile sort analysis (ANTHROPAC software, version 1.0).

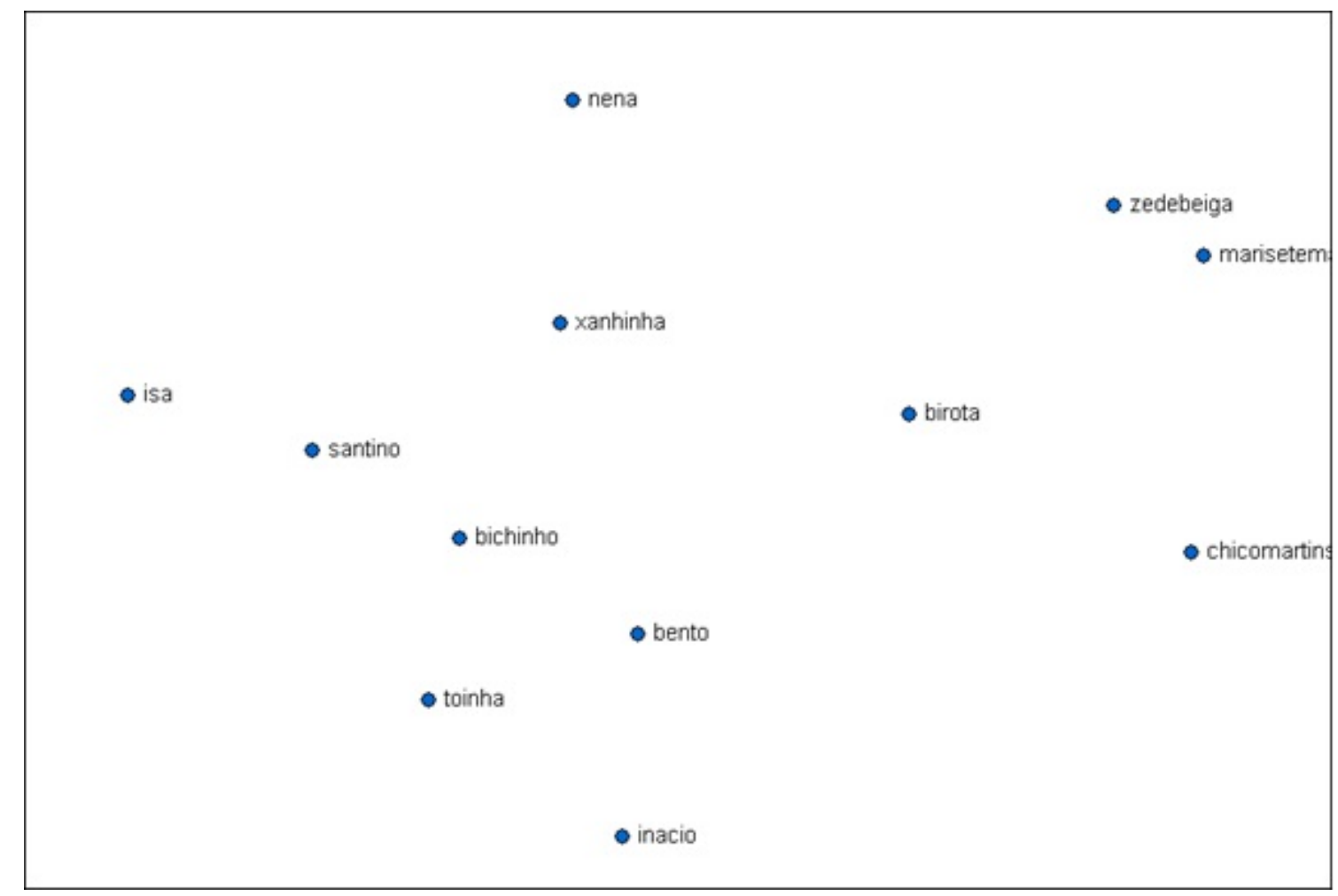

Figure 7. Grouping of local specialists in the community of Cachoeira, located in the Municipality of Soledade (Paraíba, Northeastern Brazil), using the pile sort analysis (ANTHROPAC software, version 1.0). 


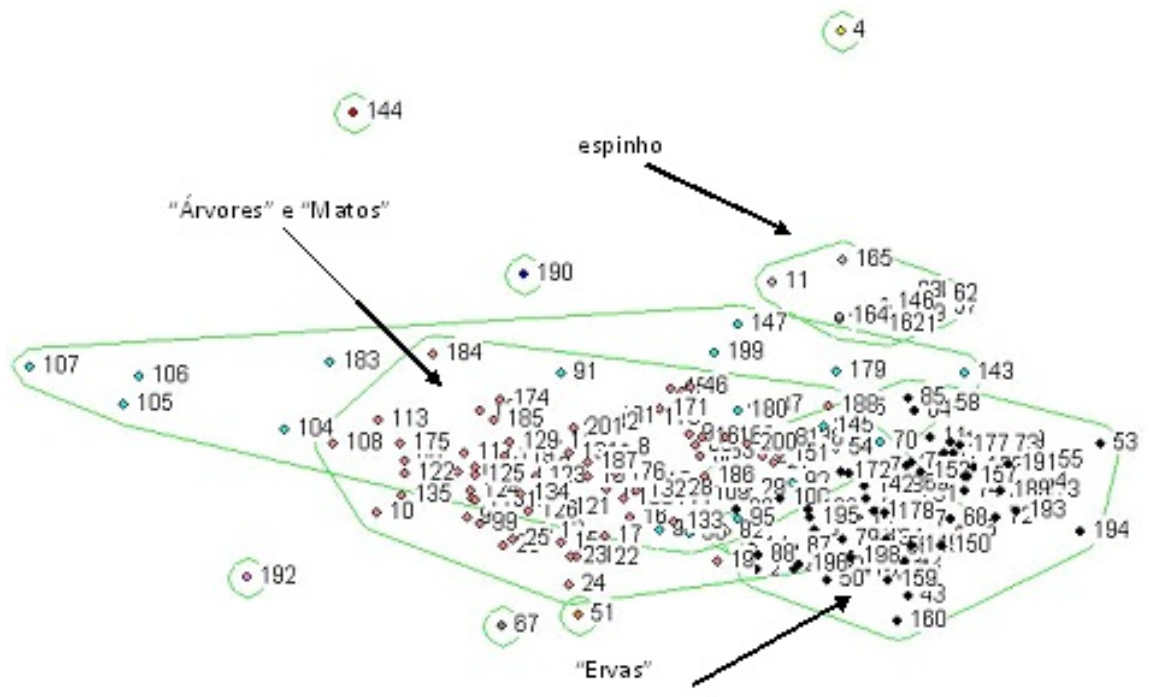

Figure 8. Grouping of folk generics, cited by local specialists in the community of Cachoeira, located in the Municipality of Soledade (Paraíba, Northeastern Brazil), in morphological groups, using the pile sort analysis (ANTHROPAC software, version 1.0).

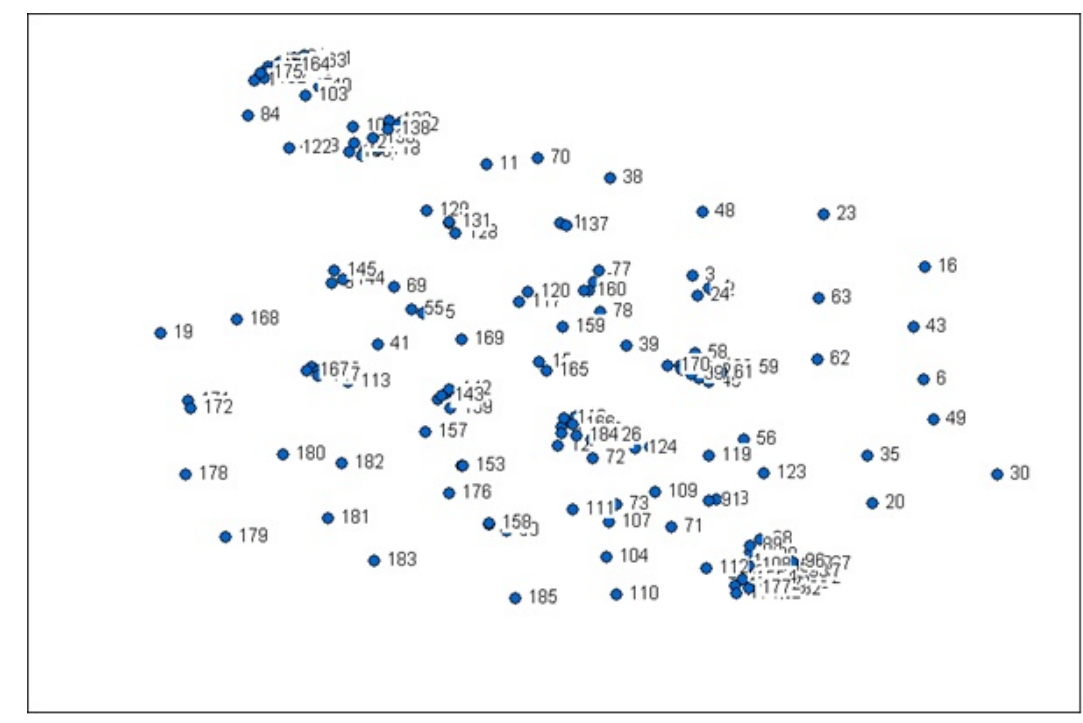

Figure 9a. Grouping of folk generics, cited by local specialists in the community of Barrocas, located in the Municipality of Soledade (Paraíba Northeastern, Brazil), in morphological groups, using the pile sort analysis (ANTHROPAC software, version 1.0). 


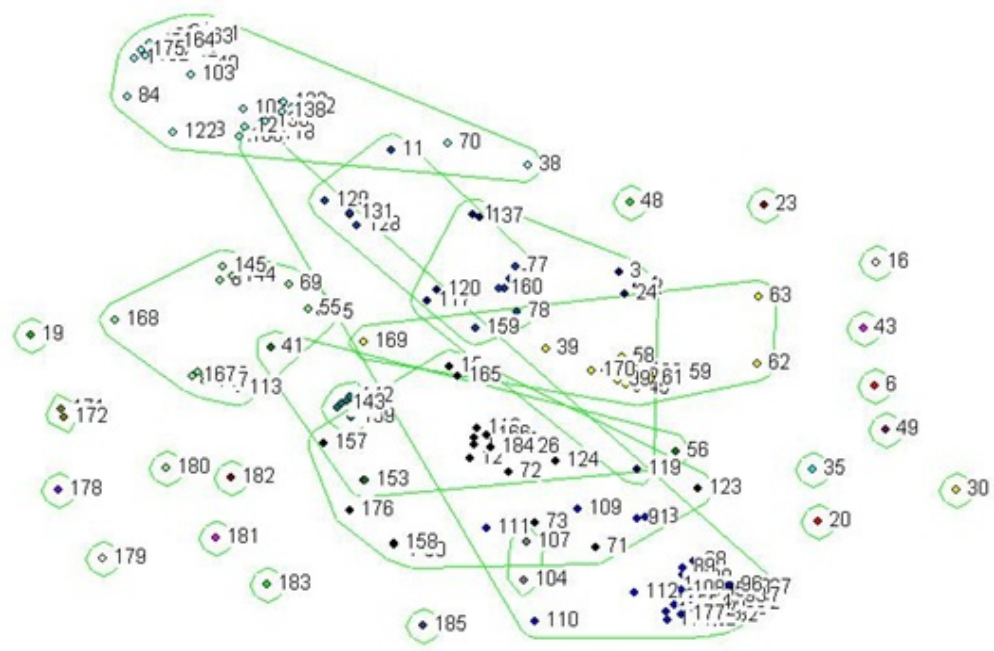

Figure 9b. Grouping of folk generics, cited by local specialists in the community of Barrocas, located in the Municipality of Soledade (Paraíba, Northeastern Brazil), in morphological groups, using the pile sort analysis (ANTHROPAC software, version 1.0).

From the grouping obtained in the pile sort analysis, it was possible to observe different situations regarding the plants indicated by Lucena et al. (2013) for conservation (Figure 10). In Barrocas, a group comprised of M. urundeuva and S. brasiliensis was isolated with no other species used for similar purposes. This makes it difficult to suggest a substitution or even management of these endangered species for others at a lower risk of local extinction because, in the local classification, the specialists indicated no other species which could share the utilitarian qualities with M. urundeuva and S. brasiliensis. Another group, formed by C. leptophloeos, A. pyrifolium, X. americana, and S. obtusifolium, remained in a position more favorable for possible indications of substitution or shared use by the local population, as these species were in a zone close to the large group of woody plants, which, from the local specialists' point of view, have similar possibilities of use, especially for timber purposes. C. blanchetianus was the only species in a position extremely favorable for reducing the exploitation pressure because it was placed in the center of the woody species group, which favors the indication of several species to replace its use. P. pyramidali stayed away from the other woody species, similar to what was observed in the first group, also making difficult the development of alternatives for sustainable use.

In the community of Cachoeira, the pile sort analysis showed groupings different from those obtained in Barrocas, because the species indicated by Lucena et al. (2013) as worthy of conservation, formed no group, remaining dispersed in the local classification, which hinders any suggestion of substitution or shared use with other species (Figure 11). 


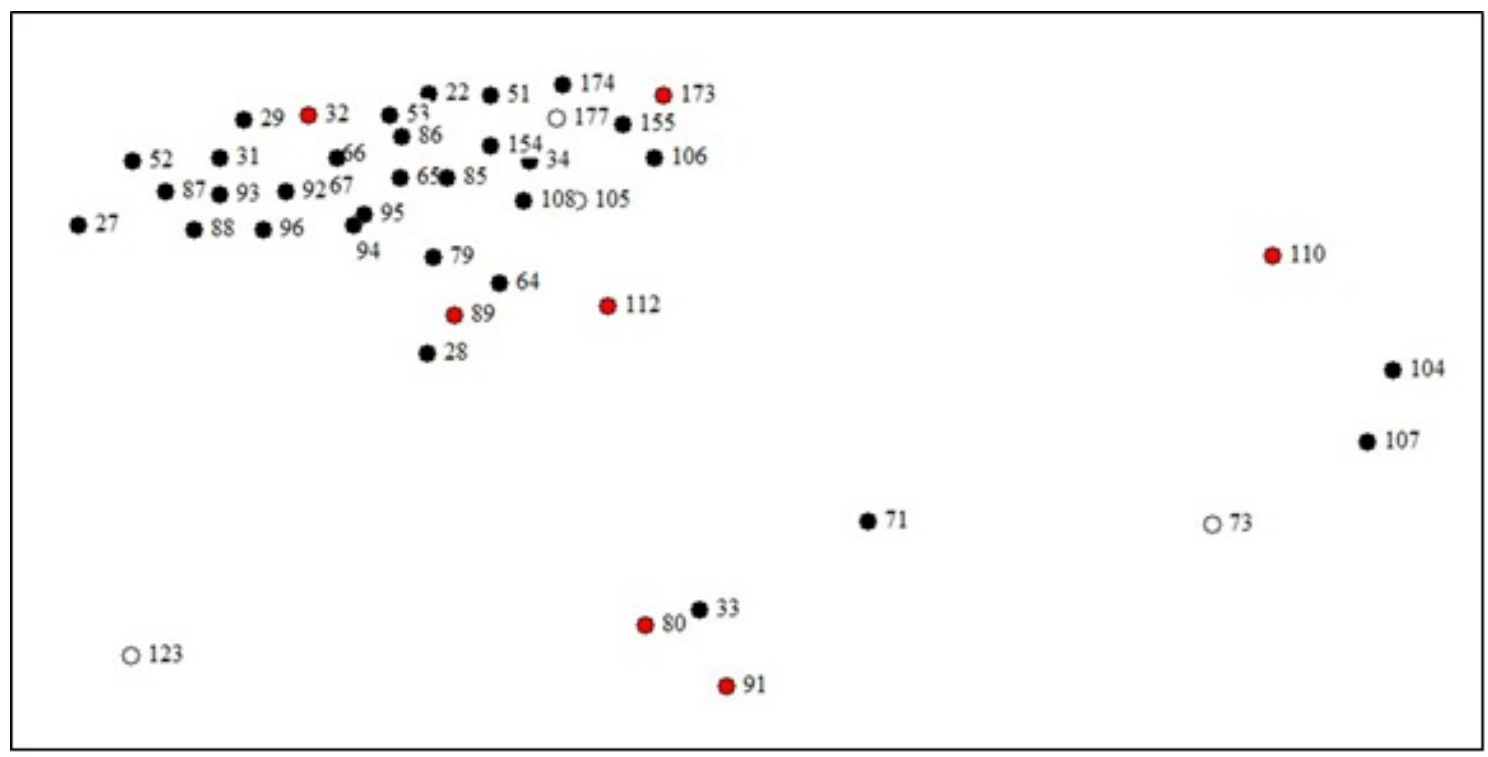

Figure 10. Grouping of woody folk generics, cited by local specialists in the community of Barrocas, located in the Municipality of Soledade (Paraíba, Northeastern Brazil), highlighted in red for species listed by Lucena et al. (2013) as priorities for conservation measures.

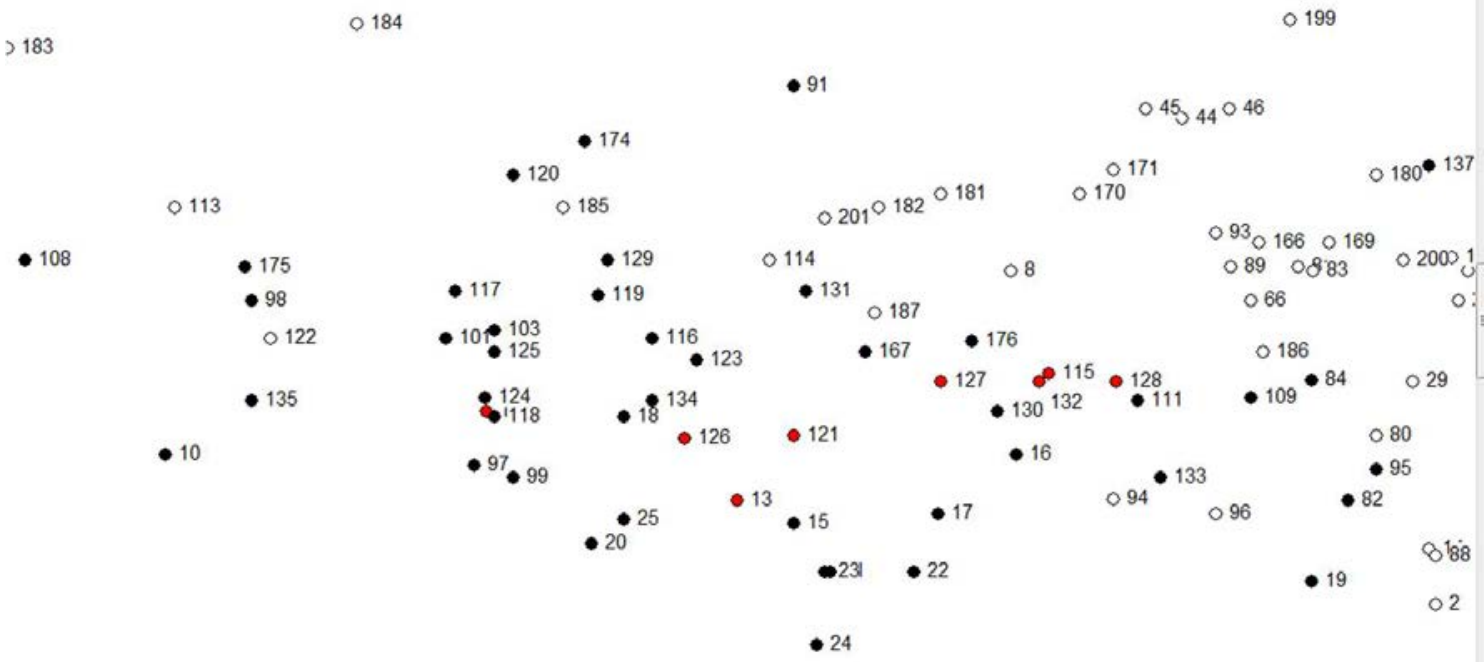

Figure 11. Grouping of woody folk generics, cited by local specialists in the community of Cachoeira, located in the Municipality of Soledade (Paraíba, Northeastern Brazil), highlighted in red for species listed by Lucena et al. (2013) as priorities for conservation measures.

\section{Discussion}

The ethnobiological classification obtained in the Municipality of Soledade may be used for rapid surveys of the local plant diversity, mainly of woody species, aiming to identify the species available in the region. In the folk classification made in this study, 65 folk generics of woody species were listed by the local specialists. Lucena et al. (2013), in a plant survey in the same region, recorded 19 woody species in Cachoeira and 29 in Barrocas. Furthermore, there was a high taxonomic correspondence of type 1:1. In other 
areas of Caatinga, vegetation surveys had different results, such as in the research by Lucena et al. (2008), who recorded 32 species in an area and 34 species in another, and in other studies by Ferraz et al. (2006), Albuquerque et al. (2005), Pereira et al. (2002), Andrade (2000), Alcoforado et al. (2003), Tavares et al. (1969), Fonseca (1991), Figueredo (2000) and Lemos and Rodal (2002), who recorded 24, 26, 37, 37, 40, 18, 25, 28, and 46 species, respectively. Comparing these results with those obtained from specialists in Soledade, it can be said that the study community has significant knowledge of species diversity from the Caatinga.

The tendency of local specialists to identify more plant categories than the botanical specialists, who have a scientific education, was also verified in field research conducted by Jinxiu et al. (2004), in China. These authors explained that the classification performed by local specialists of the ethnicity "Dai" could be used in rapid assessments of the local biodiversity; as stated in our study. This statement can be strengthened by Haverroth (2007), who studied the Kaingang indigenous people and identified in their traditional taxonomic system almost 200 folk generics, corresponding to 200 botanical species. Kakudidi (2004) emphasized that local specialists have a higher facility for identifying large-sized woody species. This ease may have contributed to the high number of woody species recorded in the present study and in that conducted by Jinxiu et al. (2004).

Berlin et al. (1973) and Berlin (1992), studying local systems of classification, proposed hierarchical ranks for comparisons with the scientific classifications. Nevertheless, this hierarchy cannot always be observed, because the folk classification might be associated with the cultural context and not only with salient characteristics of the biological resource (Ferreira-Junior et al. 2014). In Soledade, four hierarchical levels of those proposed by the abovementioned authors were identified. However, intermediate and variety levels were not found. According to Mourão et al. (2002a), only four of the six hierarchical levels are usually identified in ethnobiological classifications. Nevertheless, Hanazaki et al. (2006) found only three levels, in which "plants" and "trees", e.g., were classified at intermediate levels and the life forms had no identification. Different results were found in Soledade, where these categories were identified at the life-form level, and only the intermediate and variety levels were not identified.

The monotypic generics were the most prominent in Soledade (86\%) whereas the polytypic ones represented 14\%. This prevalence of monotypic generics has been observed in other studies such as Hays (1974), Berlin et al. (1974), Brunel (1974), Berlin (1976), Mourão and Nordi (2002a), and Farias and Alves (2007), who recorded 14\%, 16\%, $11 \%, 18 \%, 23 \%$, and $23 \%$ of polytypic generics, respectively. Berlin (1976) observed that the polytypic generics were bitypic, showing two specific taxa, as found in this study, in which all identified polytypic generics had two names.

In both study communities, morphological and utilitarian were the most prominent criteria, corroborating other studies in the literature (Berlin, 1976; Hun, 1982; Hanazaki et al., 2006; Haverroth, 2007; Pinto et al., 2016). The ecological criterion recorded in the community of Cachoeira, in our research, was also found in other studies on folk classification (Ternes et al., 2016), as well as the linguistic one (Berlin, 1976), which is used based on similarities between plant names or animal names.

The data obtained in this study emphasize the importance of knowing about the dynamic of use of plant species by the residents, due to the high number of plant utilitarian aspects used to organize their classifications. On average, $80 \%$ of the specialists highlighted such aspects in both communities. This organization based on utilitarian characteristics was defended by Hunn (1982) and Ellen (1993), who explained that the classification is specifically performed for animals and plants which have some value for the population. Haverroth (2007) also found a strong utilitarian tendency in the classification made by the Kaigangs, in which plants were grouped into different 
categories of use. Another example of this trend was recorded by Pinto et al. (2013) among fishermen in Ceará, Brazil. These authors found that fish species of commercial and/or cultural importance were mentioned in a higher number of subdivisions.

In our study, plant species were also classified by local specialists in a "nonutilitarian" category, forming a specific group. This concept of "non-useful" plants was found by Berlin (1976) in his study on the Aguaruna indigenous people, in which one-third of the known plants had no cultural importance, generally classified as monotypic generics. Likewise, in our study, non-useful plants were also monotypic generics.

In the ethnobiological classification performed in this study, species of different families were grouped in the same group, such as Poaceae and Sterculiaceae, which were classified in the same group due to their fodder potential. This was also observed for morphological characteristics, such as in the case of Sapotaceae and Mimosaceae species, which shared the same group. This trend shows that the informants follow their classificatory logic, which, in this case, differs from the scientific classification, as stated by Kakudidi (2004).

In the local classification, some groups were formed based on more than one criterion: one for a larger group and another for dividing such group into smaller groups. For example, the informants gathered several herbaceous species of different families in a huge group, using as criterion their use in the local medicine, and when asked if this group could be divided, the local specialist used the folk generics to form sub-groups based on height as a classification criterion. Similarly, "Dai" ethnicity members, in China, use varied criteria in their classification, in which the same species could receive different names according to their characteristics. Allium fistulosum, e.g., classified based on leaf size, received different names. According to the use category, Alstonia seholaris also received different names, one for medicinal and another for timber purposes. In the case of Raphanus sativus, its name changed according to the color of its flowers. However, the authors did not specify the vernacular names attributed to these species. This taxonomy classification was not performed by the residents of Soledade.

Carvalho et al. (2018) also recorded some variations in folk taxonomy made by fishermen in Rio Grande do Norte, Brazil. In their study, two shark species (Rhizoprionodon lalandii and R. porosus) were named as "cação rabo-seco". This diversity was observed in Soledade in the way of classifying and grouping the plants, in which the same species was considered, at the same time, a "tree" and a "thorn", such as in the case of "quixabeira", due to the presence of thorns on its stem. Kakudidi (2004) also found these differences in sisal plants (Agavaceae), which was classified in the life forms according to the presence or absence of inflorescences. Regarding aloes, the species were grouped according to their size, one moment in the life form "trees", the next as herbaceous. Differences in the way people perceive and classify plants was also recorded by Assogbadjo et al. (2008), who specifically analyzed the species known as Baobab (Adansonia digitata), in different African countries, and verified that the informants' perception of this plant changed from one country to another, as well as the criteria used to determine the types of Baobab.

Hanazaki et al. (2006) found an organization different from that observed in Soledade, despite using the same criteria (morphological and utilitarian). According to them, all cultivated species were classified in a group called "plant", and the arboreal species were divided into "trees with core" and "trees without core".

The life forms recorded in Soledade were named by local specialists based on morphological characteristics, such as height and presence of structures (e.g., thorn), and ecological criteria such as those used to identify the parasite, liana, and underground bush life forms. The use of these characteristics in the determination of life forms was reported by Kakudidi (2004). 
The life forms recorded in this research (large bush, medium bush, small bush, squat bush, underground bush, foliage, parasite, thorn, and forage) are probably the first records of this nomenclature in studies on botanical folk classification. Other studies identified different names for these life forms for the same species recorded in Soledade; however, the terms were closer to those used in academic organizations, such as shrubs, herbaceous, lianas, creepers, and epiphytes (Berlin, 1976; Jinxiu et al., 2004; Kakudidi 2004). The life form "tree" was recorded by Berlin (1976), Jinxiu et al. (2004), and Kakudidi (2004), liana by Jinxiu et al. (2004), and grass by Kakudidi (2004). Brow (1977) emphasized that in the local systems of biological classification, "trees" is the most frequent life form, followed by a class of small herbaceous. This justifies the diversity of life forms related to arboreal species in the current study.

In this study, the pile sort analysis showed the local specialists tend to gather plants according to their criteria, with no agreement among them. Another interesting fact in our study is that the folk classification was performed by specialists who had kinship degree, as four couples and a father with his son, residents of Cachoeira, participated in this research; however, they performed different classifications. The most similar classification was made by a couple who used similar criteria to group the plants. In Barrocas, the informants had no direct kinship. The way the local specialists used the classification criteria might have contributed to the wide universe of life form names because different life forms were recorded for arboreal plants (trees, large bush, and medium bush), herbaceous (grass, squat bush, and small bush), cacti and thorny plants (life form "thorn"), tuberous species (foliage and underground bush), and creepers and epiphytes (liana and parasite).

The groupings performed in Cachoeira tended to form the following groups: (1) woody plants, (2) cacti and bromeliads, (3) well known herbaceous, and (4) little known herbaceous. This same tendency was observed in Barrocas; however, the herbaceous were distributed into smaller and spread groups, which were organized based on utilitarian characteristics. Both for Cachoeira and Barrocas, it was found scattered species, away from the groups formed, mostly represented by exotic herbaceous species used to decorate homes, such as "rainha" (No.144), "arruda da folha azul" (No. 51), and "neuvalgina" (No. 4), in Cachoeira, and "coração de homem" (No. 23), "tambor" (No. 178), and "Benedita" (No. 185), in Barrocas.

There was a consistent relationship between the groupings made in Soledade, regarding the utilitarian and morphological characteristics, indicating the need to consider these two forms as those eventually used by human population to mentally organize the "plants' world": one involving local importance aspects of a species through its use (utilitarian or materialistic chain) and another used to organize the plants' world due to a desire to perform an intellectual systematization (not necessarily academic) of the environment in which the social group lives (cognitive and idealist chain). Hunn (1982) and Ellen (1993) consistently defend the predominance of utilitarian characteristics as a guiding criterion for biological classifications adopted by local or traditional populations. On the other hand, Levi-Strauss (1989) and Berlin (1992) defend that the classification is mainly based on cognitive aspects. However, our study showed that both aspects (intellectual and practical) may be used, in a combined way, in plant classification systems adopted locally by a certain rural human population.

\section{Ethnotaxonomic studies and local conservation}

Ethnotaxonomic research has been neglected as a methodological tool in studies focused on local biodiversity conservation, and the classifications found in the literature have mainly sought to understand the dynamic between people and animals (Mourão and Nordi, 2002a,b; Mourão et al., 2006; Farias and Alves, 2007; Souza and Begossi, 2007) or between people and plants, and generally address no conservation considerations. 
However, some authors have different approaches. Jinxiu et al. (2004) indicated the importance of the ethnotaxonomic classification for actions focused on local conservation, suggesting that it can be used in rapid biodiversity surveys. Assogbadjo et al. (2008) used the ethnotaxonomic research for a better understanding of the relationship between the informants and a single species, observing its use and possible indications for its conservation. Silva et al. (2017) used the folk classification to show that people name landscapes based on current and past use, finding an efficient strategy to optimize the use of these landscapes by the local population.

A relevant finding regarding the folk classification under study is that, in both communities and by all local specialists, it was guided by the utilitarian criterion, according to which the uses of plants were a central indicator for specialists to realize and organize the whole list of plants they knew. This fact may evidence a strong dependence these people have on plant resources, available in the region. Understanding this dependence may be important for the identification of species under a possible pressure of use, which may require attention and conservation strategies. In a study on useful plants conducted in the same communities, Lucena et al. (2013) indicated A. pyrifolium (pereiro), P. pyramidalis (catingueira), C. leptophloeos (umburana), C. blanchetianus (marmeleiro), M. urundeuva (aroeira), S. obtusifolium (quixabeira), X. americana (ameixa), and $S$. brasiliensis (baraúna), e.g., as priorities for conservation.

Analyzing how the informants distributed these species within the classification groups, both in Barrocas and Cachoeira, it was observed an interesting scenario to indicate conservation measures for such species. In Barrocas, e.g., considering only the grouping of woody plants, the species indicated by Lucena et al. (2013) as priorities for conservation were placed at the border of the group, such as in the case of S. obtusifolium, X. americana, C. leptophloeos, and A. pyrifolium. M. urundeuva and S. brasiliensis formed a group apart, and $C$. pyramidalis was isolated and away from the other groups. Only $C$. blanchetianus was located closer to the center of the general group. This scenario seems to indicate a trend toward constant and isolated uses of these species, because assuming that the utilitarian criterion was strongly used in the classifications made by the residents, it was expected that other plants with utilitarian characteristics, similar to those indicated as priorities for conservation, would be close to each other forming a group and sharing utilities. The fact that some species remained at the edge of the group and others away in a more concentrated form, may suggest that they are possibly under local pressure, perhaps confirming the indication by Lucena et al. (2013).

In Cachoeira, the scenario was a little different because the woody plants were distributed in a more spaced manner, without forming groups more defined. Another interesting aspect is that the species indicated by Lucena et al. (2013), in this case, tended to form a group, as observed for $P$. pyramidalis, C. blanchetianus, A. pyrifolium, and M. urundeuva. Another group included X. americana, S. obtusifolium, and S. brasiliensis; in this case, C. leptophloeos was apart but sharing a group with Erythrina velutina Willd. These groups were formed based on the utility of these species. The first group comprised species used in constructions and as fuel, the second group included those use in local medicine, and the third one was formed base on technological purposes. In Cachoeira, differently from Barrocas, there was a tendency toward a distribution based on plant uses, because the species were grouped according to their uses, close to other species used for similar purposes but different from those indicated by Lucena et al. (2013).

This comparative study on local forms of plant classification proved to be an effective tool for ethnobotanical studies focused on conservation measures, as it allows us to understand, partly, the dynamic of species indicated as priorities for conservation, by understanding how the informants identified and organized such species.

The hierarchical classification levels followed those indicated in the literature, being each one well defined by the informants. The life forms were abundant. Some of 
them had already been recorded in the literature and others were identified for the first time. Morphological and utilitarian were the most used classification criteria. The informants followed no consensus model in the organization of their classification.

\section{Acknocknowledgements}

The authors thank the informants of the study rural communities (Barrocas and Cachoeira) for their participation in the research.

\section{Conflict of interests}

The authors declare that they have no conflict of interests.

\section{References}

Albuquerque, U. P.; Lucena, R. F. P. Métodos e técnicas para a coleta de dados. In: Albuquerque, U. P.; Lucena, R. F. P. (Orgs.). Métodos e técnicas na pesquisa etnobotânica. Recife: NUPEEA, 2004. p. 37-62.

Albuquerque, U. P.; Andrade, L. H. C.; Silva, A. C. O. Use of plant resources in a seasonal dry forest (Northeastern Brazil). Acta Botanica Brasilica, v. 19, p. 27-38, 2005. https://doi.org/10.1590/S0102-33062005000100004

Alcoforado, F. G.; Sampaio, E. V. S. B.; Rodal, M. J. N. Florística e fitossociologia de um remanescente de vegetação caducifólia espinhosa arbórea em Caruaru, Pernambuco. Acta Botanica Brasilica, v.17, p. 287-303, 2003. 2003. https://doi.org/10.1590/S010233062003000200011

Amorim, D. S. Elementos básicos de sistemática filogenática. Ribeirão Preto: Holos Editora, 1997.

Andrade, W. M. Variações de abundância em populações de plantas da caatinga. Recife: Universidade Federal Rural de Pernambuco, 2000. (Dissertação de mestrado).

Aparicio, J. C.; Costa-Neto, E. M.; Araújo, G. P. Etnotaxonomía mixteca de algunos insectos en el municipio de San Miguel el Grande, Oxalaca, México. Etnobiología, v. 16, no. 2, p. 5875, 2018.

Araújo, H. F. P. Composição da avifauna e etnoornitologia em complexos estuariosmanguezais no Estado da Paraíba - Brasil. João Pessoa: Universidade Federal da Paraíba, 2005. (Dissertação de mestrado).

Assogbadjo, A. E.; Kakai, R. G.; Chandare, F. J.; Thomson, L.; Kyndt, T.; Sinsin, B.; Van Damme, P. Folk classification, perception, and preferences of Baobab products in West Africa: Consequences for species conservation and improvement. Economic Botany, v. 62, p. 74-84, 2008. https://doi.org/10.1007/s12231-007-9003-6

Atlas Geográfico do Estado da Paraíba. 1985.

Atran, S. Folk biology and the anthropology science: cognitive universals and cultural particulars. Behavioral and Brain Sciences, v. 21, p. 547-609, 1998. https://doi.org/10.1017/s0140525x98001277

Berlin, B. Folk systematics in relation to biological classification and nomenclature. Annual Review of Ecology and Systematics, v. 4, p. 259-271, 1973. https://doi.org/ 10.1146/annurev.es.04.110173.001355

Rev. Bras. Gest. Amb. Sustent., 2021, vol. 8, n. 20, p. 1459-1488. 
Berlin, B. The concept of rank in ethnobiological classification: Some evidence from Aguaruna folk Botany. American Ethnologist, v. 3, p. 381-399, 1976.

Berlin, B. Ethnobiological classification: Principles of categorization of plants and animals in traditional societies. Princeton, USA: Princeton University Press, 1992.

Berlin, B.; Breedlove, D. E.; Raven, P. H. Covert categories and folk taxonomies. American Anthropologist, v. 70, p. 290-299, 1968. https://doi.org/10.1525/aa.1968.70.2.02a00050

Berlin, B.; Breedlove, D. E.; Raven, P. H. Principles of Tzeltal Plant Classification: An introduction to the botanical ethnography of a Mayan-Speaking Community in Highland Chiapas. New York: Academic Press, 1974.

Braga, F.; Alves, R. R. N.; Mota, H. Sistema de classificação da mastofauna utilizados pelas comunidades locais do Parque Nacional da Quiçama, Angola. Ethnoscientia, v. 2, p. 1-10, 2017. https://doi.org/10.18542/ethnoscientia.v2i1.10186

Brown, C. H. Folk botanical life-forms: Their universality and growth. American Anthropology, v. 79, no. 2, p. 317-342, 1977.

Brunel, G. Variation in Quechua Folk Biology. Berkeley: University of California, 1974. (Ph.D. dissertation).

Bulmer, R. N. H. Folk biology in the New Guinea highlands. Social Science Information, v. 13, no. 4/5, p. 9-28, 1974. https://doi.org/10.1177\%2F053901847401300402

Carrara, E. Tsi tewara: um vôo sobre o Cerrado Xavante. São Paulo: Universidade de São Paulo, 1997. (Dissertação de mestrado).

Carvalho, M. M.; Oliveira, M. R.; Lopes, P. F. M.; Oliveira, J. E. L. Ethnotaxonomy of sharks from tropical waters of Brazil. Journal of Ethnobiology and Ethnomedicine, v. 14, no. 71, p. 1-11, 2018. https://doi.org/10.1186/s13002-018-0273-0

Clément, D. Why is taxonomy utilitarian? Journal of Ethnobiology, v. 15, no. 1, p. 1-44, 1995.

Ellen, R. The cultural relations of classifications. Cambridge: Cambridge University Press, 1993.

Farias, G. B.; Alves, A. G. C. Nomenclatura e classificação etnoornitológica em fragmentos de Mata Atlântica na Região Metropolitana do Recife, Pernambuco. Revista Brasileira de Ornitologia, v. 15, no. 3, p. 358-366, 2007.

Ferraz, J. S. F.; Albuquerque, U. P.; Meunier, I. M. J. Valor do uso e estrutura da vegetação lenhosa às margens do Riacho do Navio, Floresta, PE, Brasil. Acta Botanica Brasilica, v. 20, p. 25-134, 2006. https://doi.org/10.1590/S0102-33062006000100012

Ferreira-Junior, W. S.; Lucena, R. F. P.; Albuquerque, U. P. Visões alternativas sobre a classificação folk. In: Albuquerque, U. P. (Org.). Introdução à Etnobiologia. Recife: NUPEEA, 2014. p. 83-87.

Figueiredo, L. S. Influência dos sítios de estabelecimentos na forma das plantas de populações simpátricas da Caatinga. Recife: Universidade Federal Rural de Pernambuco, 2000. (Dissertação de mestrado).

Fonseca, M. R. Análise da vegetação arbustiva-arbórea da caatinga hiperxerófila do noroeste do Estado de Sergipe. Campinas: Universidade Estadual de Campinas, 1991. (Dissertação de mestrado). 
Hanazaki, N.; Souza, V. C.; Rodrigues, R. R. Ethnobotany of rural people from the boundaries of Carlos Botelho State Park, São Paulo State, Brazil. Acta Botanica Brasilica, v. 20, no. 4, p. 899-909, 2006. https://doi.org/10.1590/S0102-33062006000400014

Haverroth, M. Etnobotânica, uso e classificação dos vegetais pelos Kaingang. Recife, NUPEEA, 2007. (Série Estudos e Debates, 3).

Hays, T. E. Mauna: Explorations in Ndumba Ethnobotany. Washington: University of Washington, 1974. (Ph.D. dissertation).

Hays, T. E. Ndumba folk biology and general principles of ethnobotanical classification and $\begin{array}{lllll}\text { nomenclature. American Anthropologist, } & \text { v. 85, p. 592-611, }\end{array}$ https://doi.org/10.1525/aa.1983.85.3.02a00050

Hennig, W. Phylogenetic Systematic. Urbana: University of Illinois Press, 1996.

Hunn, E. The utilitarian factor in folk biological classification. American Anthropologist, v. 84, p. 830-847, 1982. https://doi.org/10.1525/aa.1982.84.4.02a00070

Jinxiu, W.; Hongmao, L.; Huabin, H.; Lei, G. Participatory approach for rapid assessment of plant diversity through a folk classification system in a Tropical Rainforest: Case study in Xishuangbanna, China. Conservation Biology, v. 18, no. 4, p.1139-1142, 2004. https://doi.org/10.1111/j.1523-1739.2004.00075.x

Kakudidi, E. K. Folk plants classification by communities around Kibale National Park, Western Uganda. African Journal of Ecology, v. 42, no.s1, p. 57-63, 2004. https://doi.org/10.1111/j.1365-2028.2004.00462.x

Lemos, R. J.; Rodal, M. J. N. Fitossociologia do componente lenhoso de um trecho da vegetação de caatinga no Parque Nacional Serra da Capivara, Piauí, Brasil. Acta Botanica Brasilica, v. 16, p. 23-42, 2002. https://doi.org/10.1590/S0102-33062002000100005

Levi-Strauss, C. 0 pensamento selvagem. Campinas: Papirus, 1989.

Lucena, R. F. P.; Lucena, C. M.; Araújo, E. L.; Alves, Â. G. C.; Albuquerque, U. P. Conservation priorities of useful plants from different techniques of collection and analysis of ethnobotanical data. Anais da Academia Brasileira de Ciências, v. 85, no. 1, p. 169-186, 2013. https://doi.org/10.1590/S0001-37652013005000013

Lucena, R. F. P.; Nascimento, V. T.; Araújo, E. L.; Albuquerque, U. P. Local uses of native plants in an area of caatinga vegetation (Pernambuco, NE-Brazil). Ethnobotany Research and Application, v. 6, p. 3-13, 2008.

Marques, J. G. W. Pescando pescadores: ciência e etnociência em uma perspectiva ecológica. 2. ed. São Paulo: NUPAUB/Fundação Ford, 2001.

Mourão, J. S. Classificação e ecologia de peixes estuarinos por pescadores do Estuário do Rio Mamanguape-PB. São Carlos: Universidade Federal de São Carlos, 2000. (Tese de doutorado).

Mourão, J. S.; Araújo, H. F. P.; Almeida, F. S. Ethnotaxonomy of mastofauna as practised by hunters of the Municipality of Paulista, State of Paraíba-Brazil. Journal of Ethnobiology and Ethnomedicine, v. 2, no. 19, p. 1-7, 2006. https://doi.org/10.1186/1746-4269-2-19

Mourão, J. S.; Montenegro, S. C. S. Pescadores e peixes: o conhecimento local e o uso da taxonomia folk baseado no Modelo Berlineano. Recife: NUPEEA. 2006. (Série Estudos e Debates, 2).

Mourão, J. S.; Nordi, N. Comparação entre as taxonomias folk e científica para peixes do Estuário do Rio Mamaguape, Paraíba - Brasil. Interciência, v. 27, p. 664-668, 2002a. 
Mourão, J. S.; Nordi, N. Pescadores, peixes, espaço e tempo: uma abordagem etnoecológica. Interciência, v. 31, no. 5, p. 358-363, 2006.

Mourão, J. S.; Nordi, N. Principais critérios utilizados por pescadores artesanais na taxonomia folk dos peixes do Estuário do Rio Mamaguape, Paraíba - Brasil. Interciência, v. 27 , no. 11 , p. 1-6, 2002 b.

Pereira, I. M.; Andrade, L. A.; Barbosa, M. R.; Sampaio, E. V. S. B. Composição florística e análise fitossociológica do componente arbustivo arbóreo de um remanescente florestal no Agreste Paraibano. Acta Botanica Brasilica, v. 16, p. 357-369, 2002. https://doi.org/10.1590/S0102-33062002000300009

Pinto, M. F.; Mourão, J. S.; Alves, R. R. N. How do artisanal fishermen name fish? An ethnotaxonomic study in Northeastern Brazil. Journal of Ethnobiology, v. 36, no. 2, p. 348-381, 2016. https://doi.org/10.2993/0278-0771-36.2.348

Pinto, M. F.; Mourão, J. S.; Alves, R. R. N. Ethnotaxonomical considerations and usage of ichthyofauna in a fishing Community in Ceará State, Northeast Brazil. Journal of Ethnobiology and Ethnomedicine, v. 9, no. 17, p.1-11, 2013. https://doi.org/10.1186/1746-4269-9-17

SEBRAE-PB - Serviço Brasileiro de Apoio às Micro e Pequenas Empresas na Paraíba. Soledade. João Pessoa: SEBRAE-PB, 1998. (Série Diagnóstico Sócio-Econômico).

Silva, T. C.; Medeiros, M. F. T.; Peroni, N.; Albuquerque, U. P. Folk classification as evidence of transformed landscapes and adaptative strategies: A case study in the semiarid region of Northeastern Brazil. Landscape Research, v. 42, no. 5, p. 521-532, 2017. https://doi.org/10.1080/01426397.2016.1258047

Souza, S. P.; Begossi, A. Whales, dolphins or fishes? The ethnotaxonomy of cetaceans in São Sebastião, Brazil. Journal of Ethnobiology and Ethnomedicine, v. 3, no. 9, p. 1-15, 2007. https://doi.org/10.1186/1746-4269-3-9

Tavares, S.; Paiva, F. A. F.; Tavares, E. J. S.; Lima, J. L. S. Inventário florestal do Ceará: estudo preliminar das matas remanescentes do município de Quixadá. Boletim de Recursos Naturais, v. 1, p. 93-111, 1969.

Ternes, M. L. F.; Gerhardinger, L. C.; Schiavetti, A. Seahorses in focus: Local ecological knowledge of seahorse-watching operators in a tropical estuary. Journal of Ethnobiology and Ethnomedicine, v. 12, no. 52, p. 1-12, 2016. https://doi.org/10.1186/s13002-0160125-8

License information: This is an open-access article distributed under the terms of the Creative Commons Attribution License, which permits unrestricted use, distribution, and reproduction in any medium, provided the original work is properly cited. 